\title{
Shock propagation through a bubbly liquid in a deformable tube
}

\author{
KEITA ANDO†, T. SANADA , K. INABAף, \\ J. S. DAMAZO, J. E. SHEPHERD, T. COLONIUS \\ AND C. E. BRENNEN \\ Division of Engineering and Applied Science, California Institute of Technology, \\ Pasadena, CA 91125, USA
}

(Received 16 February 2010; revised 8 October 2010; accepted 28 October 2010; first published online 15 February 2011)

\begin{abstract}
Shock propagation through a bubbly liquid contained in a deformable tube is considered. Quasi-one-dimensional mixture-averaged flow equations that include fluid-structure interaction are formulated. The steady shock relations are derived and the nonlinear effect due to the gas-phase compressibility is examined. Experiments are conducted in which a free-falling steel projectile impacts the top of an air/water mixture in a polycarbonate tube, and stress waves in the tube material and pressure on the tube wall are measured. The experimental data indicate that the linear theory is incapable of properly predicting the propagation speeds of finite-amplitude waves in a mixture-filled tube; the shock theory is found to more accurately estimate the measured wave speeds.
\end{abstract}

Key words: bubble dynamics, gas/liquid flows, wave-structure interactions

\section{Introduction}

Fluid-structure interaction (FSI) problems arise in industrial piping systems, underwater explosions and turbomachinery (Cole 1948; Wylie \& Streeter 1993; Brennen 1994). These flows often involve gas (or vapour) bubbles that alter the dynamics of the fluid dramatically (Brennen 1995, 2005). Dynamic loading of fluidfilled, deformable tubes has been extensively studied as an FSI model problem (Tijsseling 1996; Ghidaoui et al. 2005). Liquid-filled tubes were first studied by Korteweg (1878) and Joukowsky (1898), who introduced a linear wave speed that accounts for the compressibility of both the liquid and the structure. The KortewegJoukowsky wave speed is also known as the Moens-Korteweg wave speed in a biomedical context concerning pressure pulses through blood vessels (Pedley 1980). The wave speed in the case of bubbly liquids was later validated by Kobori, Yokoyama \& Miyashiro (1955).

$\dagger$ Present address: School of Electrical and Electronic Engineering, Nanyang Technological University, Singapore 639798. Email address for correspondence: kando@ntu.edu.sg

$¥$ Present address: Department of Mechanical Engineering, Shizuoka University, Hamamatsu 432-8561, Japan.

I Present address: Department of Mechanical Engineering and Science, Tokyo Institute of Technology, Tokyo 152-8550, Japan. 
For cases without FSI, shock problems in bubbly liquids have also been considered by many researchers. The shock theory has been validated by experiments (Campbell \& Pitcher 1958; Noordzij \& van Wijngaarden 1974; Beylich \& Gülhan 1990; Kameda $\&$ Matsumoto 1996; Kameda et al. 1998). In these experiments, bubbly mixtures were created in a tube, but the shock pressure was small enough to minimize the FSI effect. The detailed shock structure was also confirmed by computations (Kuznetsov et al. 1978; Nigmatulin, Khabeev \& Hai 1988; Watanabe \& Prosperetti 1994; Kameda \& Matsumoto 1996; Kameda et al. 1998; Delale, Nas \& Tryggvason 2005; Delale \& Tryggvason 2008). However, to the authors' knowledge, a (nonlinear) shock theory that includes both structural compressibility and bubbles has not been presented so far.

The goal of this paper is thus to develop the steady shock theory for a bubbly liquid in a deformable cylindrical tube. In $\S 2$, we describe the bubbly flow model and formulate quasi-one-dimensional equations for flows in a cylindrical tube. In $\S 3$, we introduce the propagation speed of linear waves in the mixture considering the compressibility of both the mixture and the tube, and derive the steady shock relations. In $\S 4$, we report on experiments in which a free-falling steel projectile impacts the top of a polycarbonate tube filled with air/water mixtures with void fractions up to $1 \%$. Stress waves in the tube material are measured and used to infer wave speeds. Finally, the comparison of the theory and the experiments is presented in $\S 5$.

\section{FSI bubbly flow model}

\subsection{Ensemble-averaged equations}

The liquid and disperse phases are treated as a continuum in order to evaluate the average mixture dynamics. We first review the continuum bubbly flow model (Ando 2010) based on the ensemble-averaging technique of Zhang \& Prosperetti (1994). The continuum model assumes that $(a)$ fission and coalescence of the (spherical) bubbles do not occur; $(b)$ direct interactions between the bubbles are negligible; $(c)$ wavelengths in the mixture are large compared to the average inter-bubble distance; $(d)$ the bubbles advect with the ambient liquid-phase velocity; and $(e)$ density and velocity fluctuations in the liquid phase due to the bubble oscillations are uncorrelated. The assumptions $(b)$ to $(d)$ are generally valid in the dilute limit. The model limitations are further discussed in Ando (2010).

With these assumptions, we write the one-dimensional mixture conservation equations with no FSI as

$$
\begin{aligned}
\frac{\partial \rho}{\partial t}+\frac{\partial \rho u}{\partial x} & =0 \\
\frac{\partial \rho u}{\partial t}+\frac{\partial}{\partial x}\left(\rho u^{2}+p_{l}-\tilde{p}\right) & =0 \\
\frac{\partial n}{\partial t}+\frac{\partial n u}{\partial x} & =0
\end{aligned}
$$

where $\rho$ is the mixture density, $u$ is the mixture velocity, $p_{l}$ is the averaged liquid pressure, and $n$ is the number of bubbles per unit volume of the mixture. For dilute mixtures, the mixture density is well approximated by $(1-\alpha) \rho_{l}$, where $\rho_{l}$ is the liquid density and $\alpha$ is the void fraction defined as

$$
\alpha=\frac{4 \pi}{3} n \int_{0}^{\infty} R^{3} f\left(R_{0}\right) \mathrm{d} R_{0} .
$$


Here, $R$ is the bubble radius, $R_{0}$ is the equilibrium bubble radius corresponding to the ambient pressure $p_{l 0}$, and $f\left(R_{0}\right)$ represents the normalized distribution of equilibrium bubble sizes in polydisperse mixtures. For monodisperse mixtures, the distribution is given by the Dirac delta function; the integration in (2.4) is irrelevant. Assuming that the liquid-phase flow is homentropic, the averaged liquid pressure will be described by the Tait equation of state (Thompson 1972),

$$
\frac{p_{l}+B}{p_{l 0}+B}=\frac{1}{\rho_{l 0}^{\gamma}}\left(\frac{\rho}{1-\alpha}\right)^{\gamma},
$$

where $\rho_{l 0}$ is the reference liquid density at $p_{l 0}$, and $\gamma$ and $B$ denote the stiffness and tensile strength of the liquid, respectively. The term $\tilde{p}$ in the momentum flux in (2.2) represents pressure fluctuations due to the phase interactions (Ando 2010) and vanishes in the equilibrium state. If one neglects the phase interaction term, the ensemble-averaged bubbly flow equations (2.1) to (2.3) are essentially the same as the volume-averaged equations of van Wijngaarden $(1968,1972)$.

\subsection{Quasi-one-dimensional FSI equations}

In what follows, we include the effect of FSI in the mixture-averaged equations (2.1) to (2.3). Let $A$ be the internal cross-sectional area of the cylindrical tube. We now make the following simplifications: $(f)$ the changes in the tube area are small and gradual in the flow direction; $(g)$ the tube inertia is negligible; $(h)$ the liquid pressure is only balanced by the hoop stress; and (i) the viscous shear stress on the inner wall is negligible. As a result of these assumptions, the tube area is given quasi-statically by (Shepherd \& Inaba 2009)

$$
A=A_{0}\left[1+\frac{2 a_{0}}{E h}\left(p_{l}-p_{l 0}\right)\right]
$$

where $a$ is the mid-plane tube radius that is the average of inner and outer radii, $h$ is the wall thickness, $E$ is Young's modulus of the tube material, and the subscript 0 denotes the initial (undisturbed) values. Note that the effect of $\tilde{p}$ is ignored in this expression. Also, note that in terms of hoop strains, $\epsilon_{\theta}=\left(a-a_{0}\right) / a_{0}$, the quasi-static relation (2.6) is written as

$$
\epsilon_{\theta}=\frac{a_{0}}{E h}\left(p_{l}-p_{l 0}\right) .
$$

With a conventional control volume analysis, the quasi-one-dimensional versions of (2.1)-(2.3) (in terms of the cross-sectionally averaged quantities) become

$$
\begin{aligned}
\frac{\partial \rho A}{\partial t}+\frac{\partial \rho u A}{\partial x} & =0, \\
\frac{\partial \rho u A}{\partial t}+\frac{\partial}{\partial x}\left[\rho u^{2} A+\left(p_{l}-\tilde{p}\right) A\right] & =p_{l} \frac{\partial A}{\partial x}, \\
\frac{\partial n A}{\partial t}+\frac{\partial n u A}{\partial x} & =0 .
\end{aligned}
$$

With the aid of (2.6), the momentum equation (2.9) is rewritten as

$$
\frac{\partial \rho u A}{\partial t}+\frac{\partial}{\partial x}\left[\rho u^{2} A+\left(p_{l}-\tilde{p}\right) A-\frac{A_{0} a_{0}}{E h} p_{l}^{2}\right]=0 .
$$




\section{Steady shock theory}

\subsection{Sonic speeds}

We first derive the sonic speeds of a bubbly liquid in a low-frequency limit. These are needed to define the shock Mach numbers. For convenience, we define the bulk modulus of the mixture, $K$, as

$$
\frac{1}{K}=\frac{1-\alpha}{K_{l}}+\frac{\alpha}{K_{g}},
$$

where $K_{l}$ and $K_{g}$ are the bulk moduli of the liquid and gas, respectively. For Tait liquids, we have $K_{l}=\gamma\left(p_{l}+B\right)$. If the effects of the vapour and the surface tension are neglected, we may take $K_{g}=\kappa p_{l}$, where $\kappa$ is the polytropic index of the gas. Note that in the low-frequency limit, the polytropic index approaches unity so that the bubbles behave isothermally (Brennen 1995). With the mixture bulk modulus (3.1), the sonic speed of the mixture (in which the bubbles behave quasi-statically) becomes

$$
c=\sqrt{\frac{K}{\rho}}=\sqrt{\frac{K_{l} / \rho}{1+\alpha\left(\frac{K_{l}}{K_{g}}-1\right)}} .
$$

In the dilute limit $(\alpha \rightarrow 0)$,

$$
c \rightarrow c_{l}=\sqrt{\frac{K_{l}}{\rho_{l}}},
$$

where $c_{l}$ is the sonic speed of the liquid alone.

We now include the effect of the structural compressibility on the mixture's sonic speed (3.2). The Korteweg-Joukowsky wave speed for the mixture may be defined and evaluated as

$$
c_{J}=\left(\left.\frac{1}{A} \frac{\partial \rho A}{\partial p_{l}}\right|_{\kappa}\right)^{-1 / 2}=\frac{c}{\sqrt{1+\xi}}=\sqrt{\frac{K_{l} / \rho}{1+\alpha\left(\frac{K_{l}}{K_{g}}-1\right)+\xi_{l}}},
$$

where $\xi$ and $\xi_{l}$ determine the extent of fluid-structure coupling for the cases of the mixture and the liquid alone, respectively:

$$
\xi=\frac{2 K a_{0}}{E h}, \quad \xi_{l}=\frac{2 K_{l} a_{0}}{E h} .
$$

This wave speed (3.4) for the mixture is identical to that of Kobori et al. (1955). It yielded good agreement with their experiments. Note that the structural compressibility reduces the linear wave speed in the mixture (i.e. $c_{J}<c$ ). In the dilute limit,

$$
c_{J} \rightarrow c_{l J}=\frac{c_{l}}{\sqrt{1+\xi_{l}}},
$$

where $c_{l J}$ is the (non-dispersive) wave speed for the case of the liquid alone.

Skalak (1956) quantified the effects of tube dynamics, with shell theory, on linear waves (also see Tijsseling et al. 2008). Even for the case of pure liquids, the tube inertia with bending resistance leads to wave dispersion. Skalak's extended theory of water hammer predicts the wave speeds, in a low-frequency limit, for the pure liquid 
case:

$$
c_{1}, c_{2}=c_{l}\left[\frac{r_{3} \mp \sqrt{r_{3}^{2}-4 r_{2}^{2}\left(1-v^{2}\right)\left(2 r_{1}+r_{2}\right)}}{2\left(2 r_{1}+r_{2}\right)}\right]^{1 / 2},
$$

where

$$
r_{1}=\frac{\rho_{l}}{\rho_{s}} \frac{a_{0}}{h}, \quad r_{2}=\frac{c_{s}^{2}}{c_{l}^{2}}, \quad r_{3}=2 r_{1} r_{2}+r_{2}+r_{2}^{2}\left(1-v^{2}\right), \quad c_{s}=\sqrt{\frac{E}{\rho_{s}\left(1-v^{2}\right)}} .
$$

Here, $c_{s}, \rho_{s}$ and $v$ are the (longitudinal) sonic speed, the density and the Poisson's ratio of the tube material, respectively. The wave speeds $c_{1}$ and $c_{2}$ are the extended versions of the Korteweg-Joukowsky wave speed $c_{l J}$ and the precursory wave speed, respectively. For the experiments presented in $\S 4$ with water $\left(c_{l}=1491 \mathrm{~m} \mathrm{~s}^{-1}\right)$ in a polycarbonate tube $\left(E=2.13 \mathrm{GPa}, \rho_{s}=1200 \mathrm{~kg} \mathrm{~m}^{-3}, v=0.37, a_{0}=3.5 h=22.2 \mathrm{~mm}\right)$, we obtain

$$
c_{l J}=517.9 \mathrm{~m} \mathrm{~s}^{-1}, \quad c_{1}=512.6 \mathrm{~m} \mathrm{~s}^{-1}, \quad c_{s}=1434 \mathrm{~m} \mathrm{~s}^{-1}, \quad c_{2}=1435 \mathrm{~m} \mathrm{~s}^{-1} .
$$

It follows that the speed $c_{1}$ is only slightly lower than the Korteweg-Joukowsky speed $c_{l J}$. Hence, in the low-frequency limit, the effects of the tube dynamics on the primary wave speed are negligible. We also notice that the precursory wave speed $c_{2}$ is very close to the sonic speed of the tube material.

\subsection{Steady shock relations}

We now develop the steady shock relations for a shock in a bubbly liquid in a deformable cylindrical tube. In front of the shock, the bubbles are in equilibrium at $\left(R_{0}, T_{0}, p_{l 0}\right)$, where $T_{0}$ is the initial temperature of the bubble contents. Far downstream of the shock front, the bubble dynamics are finally damped out and the bubbles are once again in equilibrium at $\left(R_{H}, T_{0}, p_{l H}\right)$, where $R_{H}$ is the new equilibrium radius corresponding to shock pressure $p_{l H}>p_{l 0}$. The specification of $T_{0}$ in the final equilibrium state follows from the assumption that the liquid temperature is undisturbed and the bubble temperature finally returns to the liquid temperature. Note that under equilibrium conditions, the term $\tilde{p} A$ in the momentum equation (2.11) vanishes.

The quasi-one-dimensional equations (2.8), (2.11) and (2.10) are now written in a coordinate system $\left(x^{\prime}=x-U_{s} t\right.$ and velocity $\left.u^{\prime}\right)$ moving with the shock velocity $U_{s}$ :

$$
\begin{aligned}
\frac{\mathrm{d}}{\mathrm{d} x^{\prime}}\left(\rho u^{\prime} A\right) & =0, \\
\frac{\mathrm{d}}{\mathrm{d} x^{\prime}}\left(\rho u^{\prime 2} A+g\left(p_{l}\right)-\tilde{p} A\right) & =0, \\
\frac{\mathrm{d}}{\mathrm{d} x^{\prime}}\left(n u^{\prime} A\right) & =0,
\end{aligned}
$$

where

$$
g\left(p_{l}\right)=A_{0}\left(1-\frac{2 p_{l 0} a_{0}}{E h}\right) p_{l}+\frac{A_{0} a_{0}}{E h} p_{l}^{2} .
$$

Integrating (3.10) to (3.12) from upstream (denoted by the subscript 0) to far downstream (denoted by the subscript $H$ ), it transpires that, independent of the 
detailed shock structure,

$$
\begin{aligned}
-\rho_{H} u_{H}^{\prime} A_{H} & =\rho_{0} U_{s} A_{0}, \\
\rho_{H} u_{H}^{\prime 2} A_{H}+g\left(p_{l H}\right) & =\rho_{0} U_{s}^{2} A_{0}+g\left(p_{l 0}\right), \\
-n_{H} u_{H}^{\prime} A_{H} & =n_{0} U_{s} A_{0},
\end{aligned}
$$

where $\rho_{0}=\left(1-\alpha_{0}\right) \rho_{l 0}$ and $\rho_{H}=\left(1-\alpha_{H}\right) \rho_{l H}$.

The shock pressure, $p_{l H}$, may be written as

$$
p_{l H}=\left(p_{l 0}-p_{v}+\frac{2 \Upsilon}{R_{0}}\right)\left(\frac{R_{H}}{R_{0}}\right)^{-3 \kappa}+p_{v}-\frac{2 \Upsilon}{R_{H}},
$$

where $\kappa$ is set to unity because the bubble temperature eventually returns to $T_{0}$. If vapour pressure $p_{v}$ and surface tension $\Upsilon$ are neglected, (3.17) reduces to

$$
p_{l H}=p_{l 0}\left(\frac{R_{H}}{R_{0}}\right)^{-3} \text {. }
$$

It follows from (3.14) and (3.16) that

$$
n_{H}=n_{0}\left[\left(1-\alpha_{0}\right)\left(\frac{p_{l 0}+B}{p_{l H}+B}\right)^{1 / \gamma}+\frac{4 \pi}{3} n_{0} \int_{0}^{\infty} R_{H}^{3} f\left(R_{0}\right) \mathrm{d} R_{0}\right]^{-1} .
$$

With the aid of (3.17) and (3.19), the void fraction $\alpha_{H}$ corresponding to $p_{l H}$ is computed by (2.4). With the neglect of vapour pressure and surface tension, (3.19) reduces to

$$
n_{H}=n_{0}\left[\left(1-\alpha_{0}\right)\left(\frac{p_{l 0}+B}{p_{l H}+B}\right)^{1 / \gamma}+\alpha_{0} \frac{p_{l 0}}{p_{l H}}\right]^{-1},
$$

and the void fraction at $p_{l H}$ is

$$
\alpha_{H}=\frac{4 \pi}{3} n_{H} \int_{0}^{\infty} R_{H}^{3} f\left(R_{0}\right) \mathrm{d} R_{0}=\left[1+\frac{1-\alpha_{0}}{\alpha_{0}} \frac{p_{l H}}{p_{l 0}}\left(\frac{p_{l 0}+B}{p_{l H}+B}\right)^{1 / \gamma}\right]^{-1} .
$$

In this manipulation, we do not make any assumptions regarding the shape of the gas volume so that the bubble number conservation equation (2.3) is irrelevant; the relation (3.21) simply represents the conservation of the gas-phase mass. This implies that the effect of bubble fission or coalescence may be unimportant in determining the steady shock speed if the effects of vapour pressure and surface tension are minimal (i.e. if the bubbles are not too small). This fact may be verified by the observation that the void fraction (3.21) at $p_{l H}$ does not depend explicitly on the bubble number density.

From (3.14) and (3.15), the steady shock speed becomes

$$
U_{s}=\sqrt{\frac{g\left(p_{l H}\right)-g\left(p_{l 0}\right)}{\rho_{0} A_{0}\left(1-\frac{\rho_{0} A_{0}}{\rho_{H} A_{H}}\right)}},
$$

and the induced velocity far downstream of the shock front is then given by

$$
u_{H}=u_{H}^{\prime}+U_{s}=\left(1-\frac{\rho_{0} A_{0}}{\rho_{H} A_{H}}\right) U_{s} .
$$



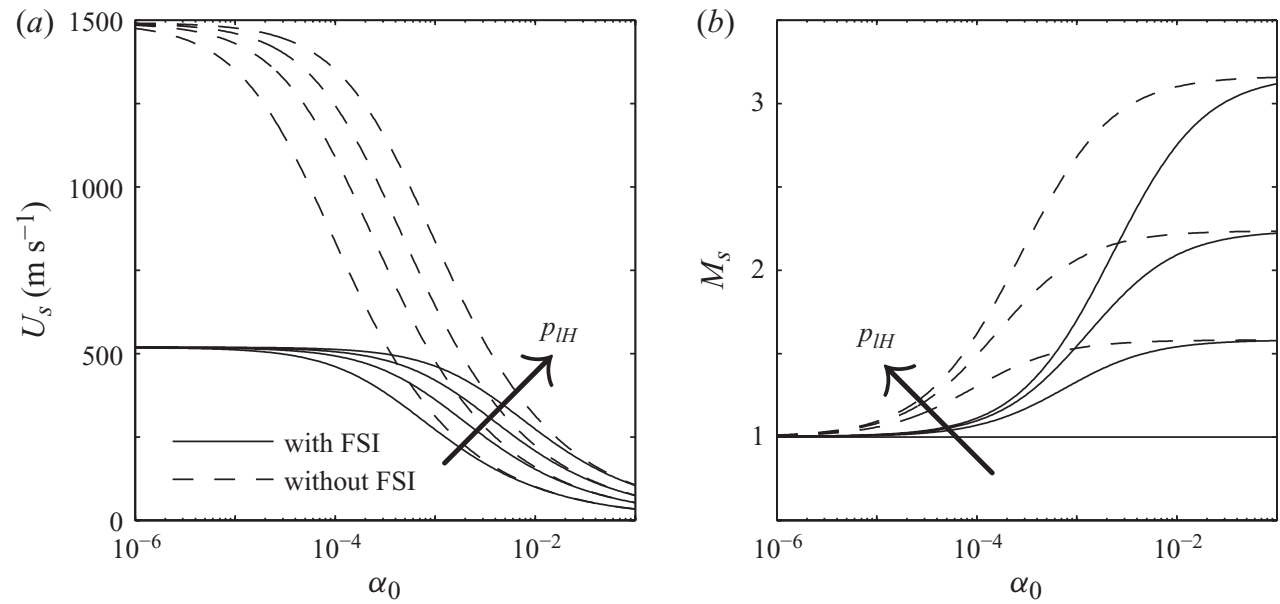

FIGURE 1. (a) Steady shock speeds and $(b)$ shock Mach numbers as a function of initial void fraction in bubbly water with $\xi_{l}=7.29$ (FSI) and $\xi_{l}=0$ (no FSI). The curves are parametrized by the shock pressure $p_{l H} / p_{l 0}=1,2.5,5,10$, where $p_{l 0}=101 \mathrm{kPa}$.

It is readily shown that the shock speed (3.22) approaches the Korteweg-Joukowsky wave speed (3.4) if the shock strength is infinitesimal. Consequently, the shock Mach number may be defined as

$$
M_{s}=\frac{U_{s}}{c_{J}} .
$$

In the limit of infinite structural stiffness $(E \rightarrow \infty)$, the steady shock relations derived in this section reduce to the standard result for dilute bubbly flows (see for example Ando 2010):

$$
U_{s}=\sqrt{\frac{p_{l H}-p_{l 0}}{\rho_{0}\left(1-\frac{\rho_{0}}{\rho_{H}}\right)}}, \quad u_{H}=\left(1-\frac{\rho_{0}}{\rho_{H}}\right) U_{s}, \quad M_{s}=\frac{U_{s}}{c} .
$$

\subsection{Gas-phase nonlinearity}

We document the steady shock relations for the case of bubbly water with $\xi_{l}=7.29$, where the value of $\xi_{l}$ is computed based on the properties of the polycarbonate tube that is used in the experiments described in $\S 4$. For simplicity, we ignore the effects of vapour pressure and surface tension. Figure 1 demonstrates the effects of the initial void fraction and the shock pressure on the shock speed and Mach number. Note that $p_{l H}=p_{l 0}$ indicates the linear wave cases, in which the shock speeds (3.22) and (3.25a) reduce to the sonic speeds (3.4) and (3.2), respectively. It follows from figure 1(a) that the gas-phase compressibility dominates over the compressibility of the water and structure for a sufficiently high void fraction. It is also seen that the shock speeds are greatly reduced by even a tiny void fraction. Moreover, unless the void fraction is extremely small, the finite shock strength yields a significant deviation from the linear wave speed due to the nonlinearity associated with the gas-phase compressibility. As a result, the shock Mach number increases as the void fraction increases, as seen in figure $1(b)$. We note that the shock Mach numbers are only slightly greater than 1 for the case of water alone $\left(\alpha_{0}=0\right)$ since the pressure perturbations (in water) up to several hundred atmospheres remain very weak (Thompson 1972). This fact 


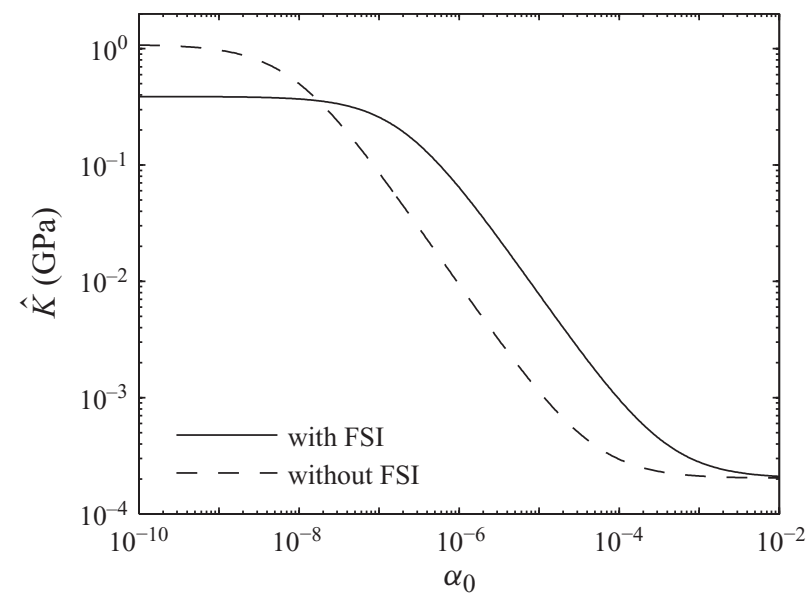

FIGURE 2. Modified bulk modulus of bubbly water with $\xi_{l}=7.29$ (FSI) and $\xi_{l}=0$ (no FSI).

was experimentally confirmed by Nagayama, Mori \& Shimada (2002) and Inaba \& Shepherd (2010).

To quantify the effect of the gas-phase nonlinearity, we further examine the steady shock relations. For the case of infinitesimal shock strength $\left(\Delta p_{l}=p_{l H}-p_{l 0} \ll p_{l 0}\right)$, the shock speed (3.22) can be approximated by

$$
U_{s} \approx c_{J}\left(1+\frac{\Delta p_{l}}{\hat{K}}\right),
$$

where $\hat{K}$ is defined as

$$
\hat{K}=c_{J}\left(\left.\frac{\mathrm{d} U_{s}}{\mathrm{~d} \Delta p_{l}}\right|_{\Delta p_{l}=0+}\right)^{-1} .
$$

In the limits of $\alpha_{0} \rightarrow 0$ (pure liquid) and $\xi \rightarrow 0$ (no FSI), we find $\hat{K} \rightarrow 4 K_{l} /(\gamma+1)$. Thus, $\hat{K}$ may be called the modified bulk modulus. It follows from (3.26) that the linear theory (where the relation $U_{s}=c_{J}$ holds) is effectively valid under the condition $\hat{K} \gg \Delta p_{l}$. Hence, the modified bulk modulus, $\hat{K}$, of the mixture can be regarded as a measure of the nonlinearity in the sense that larger values of $\hat{K}$ make the linear theory applicable to a broader range of the shock pressures. The modified bulk moduli of bubbly water (with and without FSI) are plotted as a function of $\alpha_{0}$ in figure 2. It turns out that even a small void fraction yields several-orders-of-magnitude reduction in the value of $\hat{K}$. This implies that the applicability of the linear theory is limited in the case of bubbly mixtures. In other words, unless the void fraction is extremely small, the gas-phase nonlinearity comes into play and the shock theory is needed to properly predict the wave speeds.

\section{Water-hammer experiments}

\subsection{Experimental set-up}

Experiments were conducted in order to measure wave speeds in a mixture-filled tube. The experimental apparatus depicted in figure 3 is similar to that of Inaba \& Shepherd (2010), and consists of a vertical polycarbonate tube (PCT0021.25, San Diego Plastics; $E=2.13 \mathrm{GPa}, \rho_{s}=1200 \mathrm{~kg} \mathrm{~m}^{-3}, a_{0}=3.5 \mathrm{~h}=22.2 \mathrm{~mm}$ ) filled with an air/water mixture. 


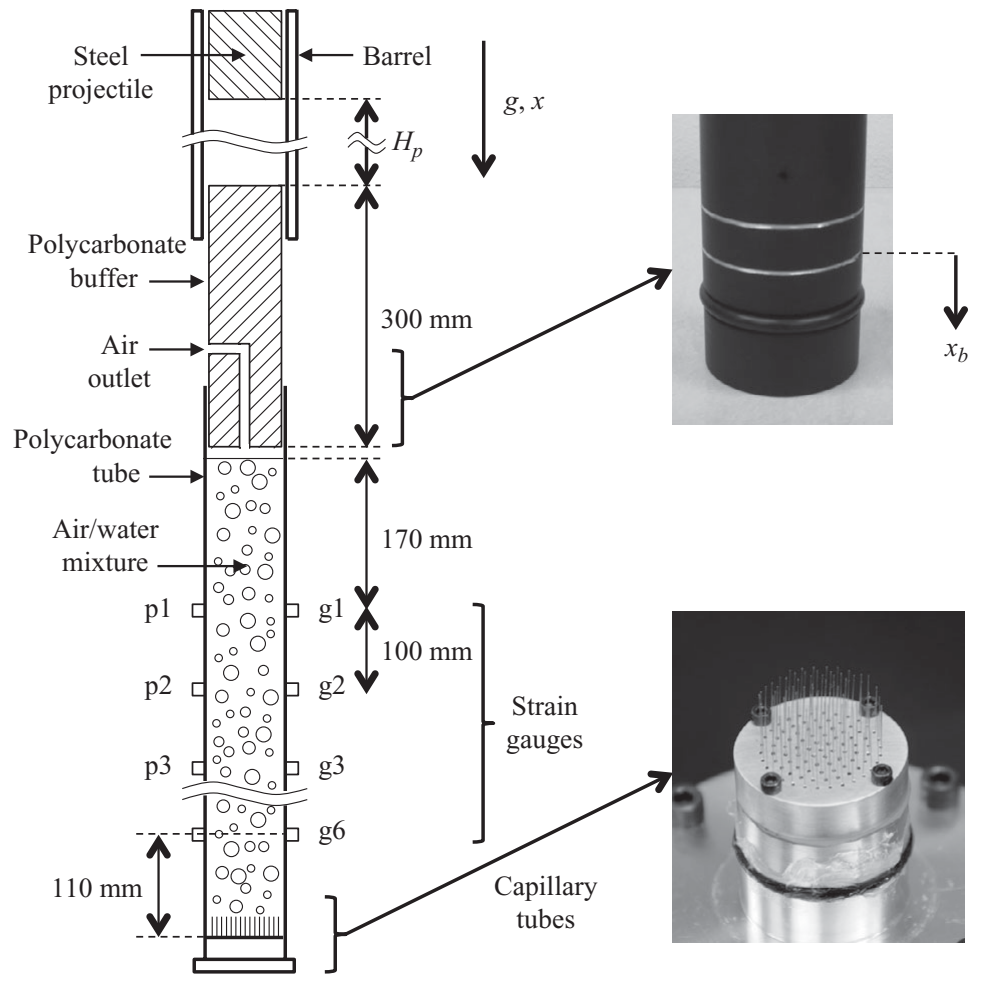

Figure 3. A schematic diagram of the experimental set-up.

A barrel is mounted above the tube and a $1.50 \mathrm{~kg}$ cylindrical steel projectile falls under gravity, $g$. The free-falling projectile (with drop height $H_{p}=2 \mathrm{~m}$ or $0.5 \mathrm{~m}$ ) impacts a $0.42 \mathrm{~kg}$ polycarbonate buffer inserted into the top of the tube rather than directly hitting the bubbly liquid surface. Stress waves in the tube are measured using six strain gauges (SR-4, Vishay; denoted by g1 to g6 in figure 3) placed at intervals of $100 \mathrm{~mm}$ along the tube and oriented in the hoop direction. The signals are processed using a signal conditioning amplifier (2300 System, Vishay) and are stored in a digital recorder (NI 6133, National Instruments; sampling rate 2.5 MHz). Strain detection at the strain gauge location g2 triggers the recording at $t=0$. For selected cases, liquid pressure on the inner tube wall is measured using six pressure transducers, $\mathrm{p} 1$ to p6 (Model 113A24, PCB Piezotronics), which are located at the opposite side of the strain gauges.

It should be pointed out that the buffer motion (before the direct impact on the fluid surface) compresses the air in the gap between the column surface and the bottom of the buffer. The wave that results from the air compression thus propagates through the fluid column before the direct collision. However, the air inertia is negligible compared with the buffer inertia, so the resulting wave will have smaller energy than that generated by the direct impact of the buffer.

\subsection{Method of bubble generation}

The bubbles are created using a bubble generator consisting of an aluminium plate and capillary tubes (TSP020150, Polymicro Technologies; inner diameter $20 \mu \mathrm{m}$ ), as shown in figure 3 . The intent is to create small bubbles and as homogeneous a mixture as possible. Up to an initial void fraction of $\alpha_{0}=0.0056,91$ capillary tubes are used; 

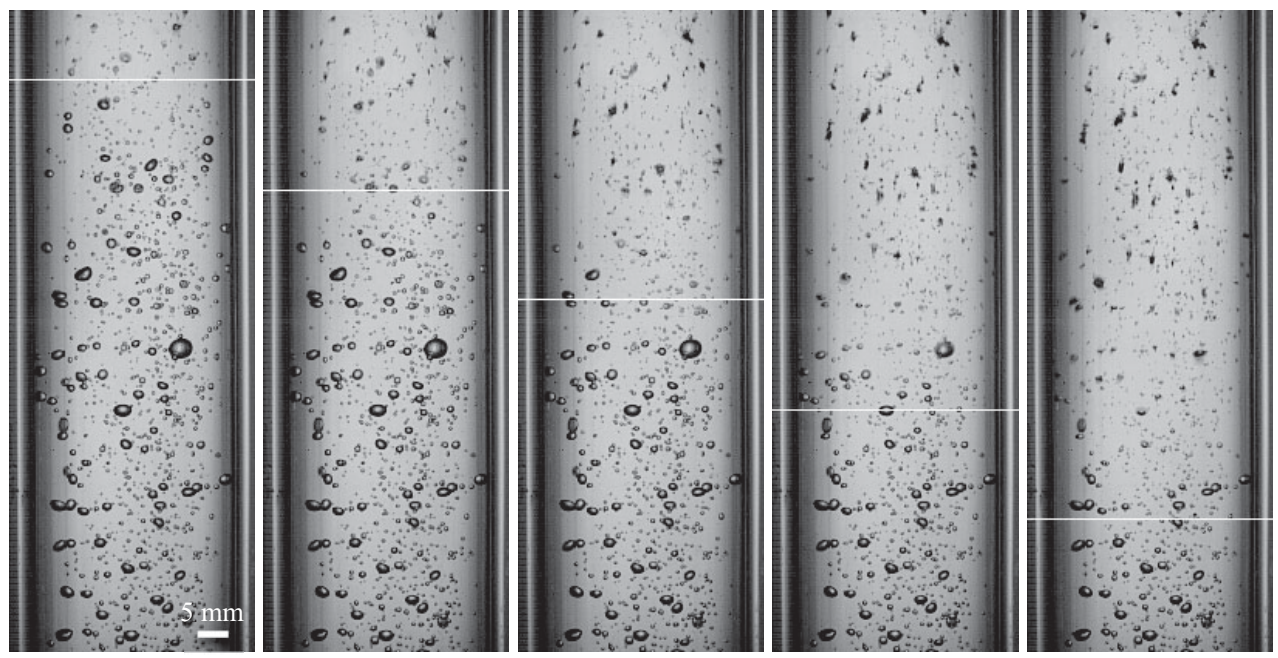

FIGURE 4. Evolution of the compression wave that propagates downward through an air/water mixture for $H_{p}=2 \mathrm{~m}$ and $\alpha_{0}=0.0081$. The white lines denote the estimated wavefront position. The frame rate is 20000 f.p.s.

for higher void fractions, the number increases to 217. The capillary tubes are located in the drilled holes of the plate and are fastened with epoxy. One side of the plate is tightly covered with a chamber. The chamber is pressurized, and the air is injected, due to the pressure head, into the fluid column. The injected bubbles rise upward to the column surface, and eventually escape from an air outlet in the buffer.

Distilled water is used for the case of no air injection; otherwise, tap water is used. Note that the volume of tiny bubbles present in tap water is negligible compared with that of the injected air. The water temperature is kept $23^{\circ} \mathrm{C}$ so that the vapour pressure $\left(p_{v}=2.8 \mathrm{kPa}\right)$ is much smaller than one atmosphere, $p_{l 0}$. The sonic speed of water, $c_{l}$, at $23^{\circ} \mathrm{C}$ is $1491 \mathrm{~m} \mathrm{~s}^{-1}$ with $\gamma=7.15$ and $B=310 \mathrm{MPa}$.

Images of the bubbles are captured by a high-speed video camera (Phantom v7.3, Vision Research). A white LED lamp (Model 900445 , Visual Instrumentation Corporation) is used for backlighting. A water jacket is attached outside the tube to minimize image distortion. The evolution of the compression wave for the case of $H_{p}=2 \mathrm{~m}$ and $\alpha_{0}=0.0081$ is shown in figure 4 , where the wavefront position is also estimated from the measured wave speed $\left(U_{s}=355 \mathrm{~m} \mathrm{~s}^{-1}\right)$ that is computed in $\S 4.6$. It turns out that the predicted wave position generally corresponds with where the bubbles are collapsing. Moreover, the bubble size is found to be broadly distributed (i.e. the mixture is polydisperse), and the mixture is nearly homogeneous. The detailed images of the compressed bubbles are presented in figure 5. The bubble fission in figure 5(a) may be due to a Rayleigh-Taylor-type instability (Brennen 2002). The re-entrant jets in figure $5(b)$ are induced by interaction with the shock wave (see for example Johnsen \& Colonius 2009).

The initial void fraction (up to $1 \%$ ) is estimated based on the difference in the column height with and without the air injection. Uncertainty in this measurement is $\pm 0.1 \mathrm{~mm}$ except for the case of the highest void fraction, $\alpha_{0}=0.01$, in which the column surface waves increase the uncertainty to $\pm 0.5 \mathrm{~mm}$. In the experiments, the following void fractions were tested: $\alpha_{0}=0$ (no air injection), $0.0013 \pm 0.0001$, $0.0024 \pm 0.0001,0.0056 \pm 0.0001,0.0081 \pm 0.0001$ and $0.010 \pm 0.001$. 
(a)

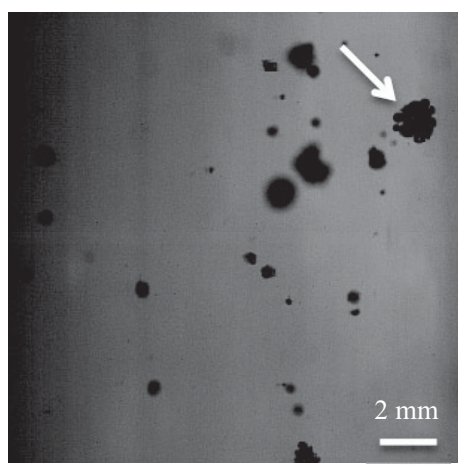

(b)

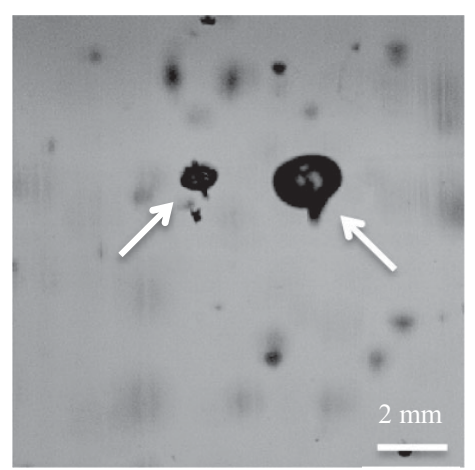

FIGURE 5. Examples of the images of $(a)$ bubble fission and $(b)$ re-entrant jets. The frame rates are 25000 and 79000 f.p.s., respectively.

(a)

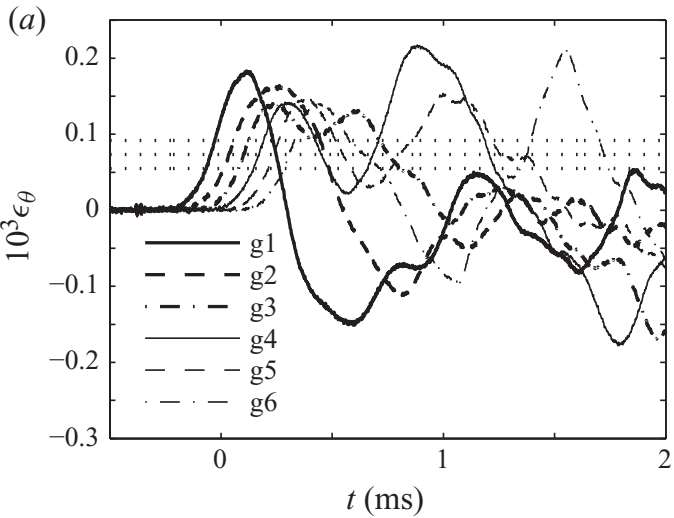

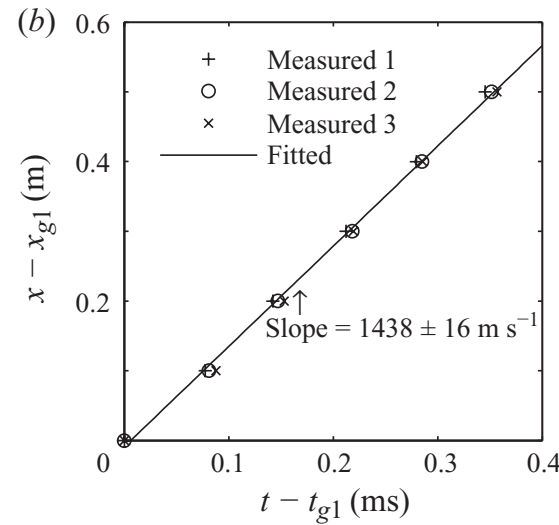

FIGURE 6. An example of $(a)$ the evolution of hoop strains without FSI and $(b)$ the locations of the wavefronts. The dotted lines in $(a)$ denote the threshold values used to determine the wavefronts for $(b)$.

\subsection{Precursory wave speeds}

In order to verify the physical properties of the tube material, stress waves were generated by hammering the top of the tube and, with no water in the tube, wave speeds were computed. The evolution of the hoop strains is presented in figure 6. For comparative purposes, three threshold strain values $(30 \%, 40 \%$ and $50 \%$ of the maximum strain measured at the strain gauge g1) are used to determine the position of the wavefront. Then, the wave speed was obtained from the slope of a linear least-squares fit to the wavefront positions; the standard deviation of the slope was also computed. It transpires that the wave speed is fairly constant and the dispersion resulting from the thresholding is very small. Furthermore, the computed wave speed is found to agree with the theoretical value of $c_{s}=1434 \mathrm{~m} \mathrm{~s}^{-1}$ discussed in $\S 3.1$. We also note that within the measurement period, the error associated with the sampling rate $( \pm 0.4 \mu \mathrm{s})$ or the inter-gauge distance $( \pm 1 \mathrm{~mm})$ is negligible compared with that of the thresholding.

We now examine the precursory waves for the tube filled with water. Figure 7 shows the precursory wave evolution and the wavefront location for $H_{p}=2 \mathrm{~m}$ and $\alpha_{0}=0$. The thresholding is again based on $30 \%, 40 \%$ and $50 \%$ of the minimum 

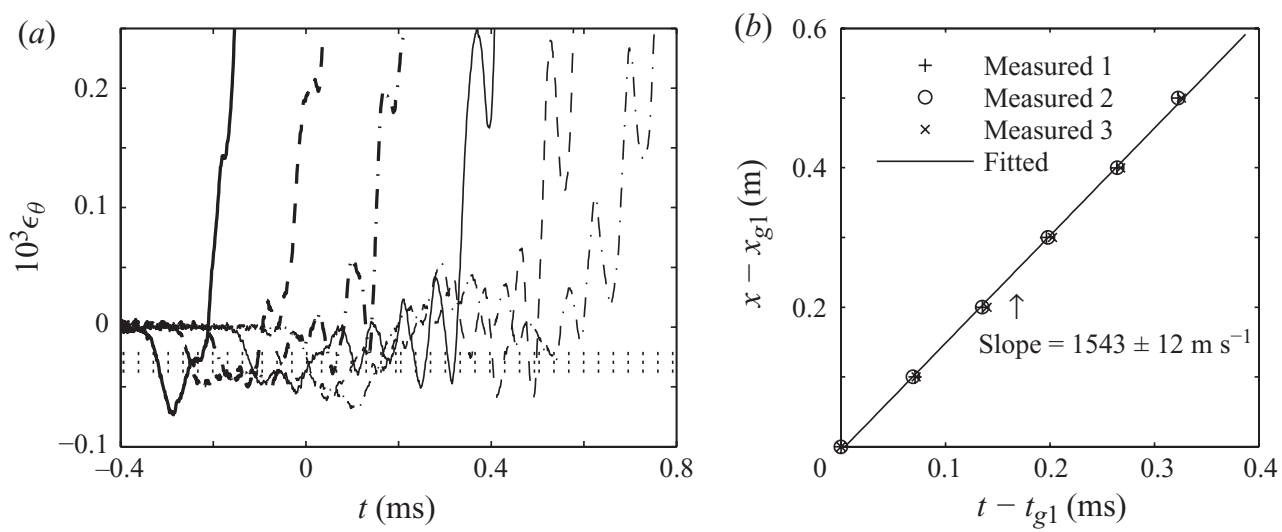

FIGURE 7. An example of $(a)$ the evolution of hoop strains with FSI and $(b)$ the locations of the precursory wavefronts for $H_{p}=2 \mathrm{~m}$ and $\alpha_{0}=0$. The dotted lines in $(a)$ denote the threshold values used to determine the wavefronts for $(b)$.

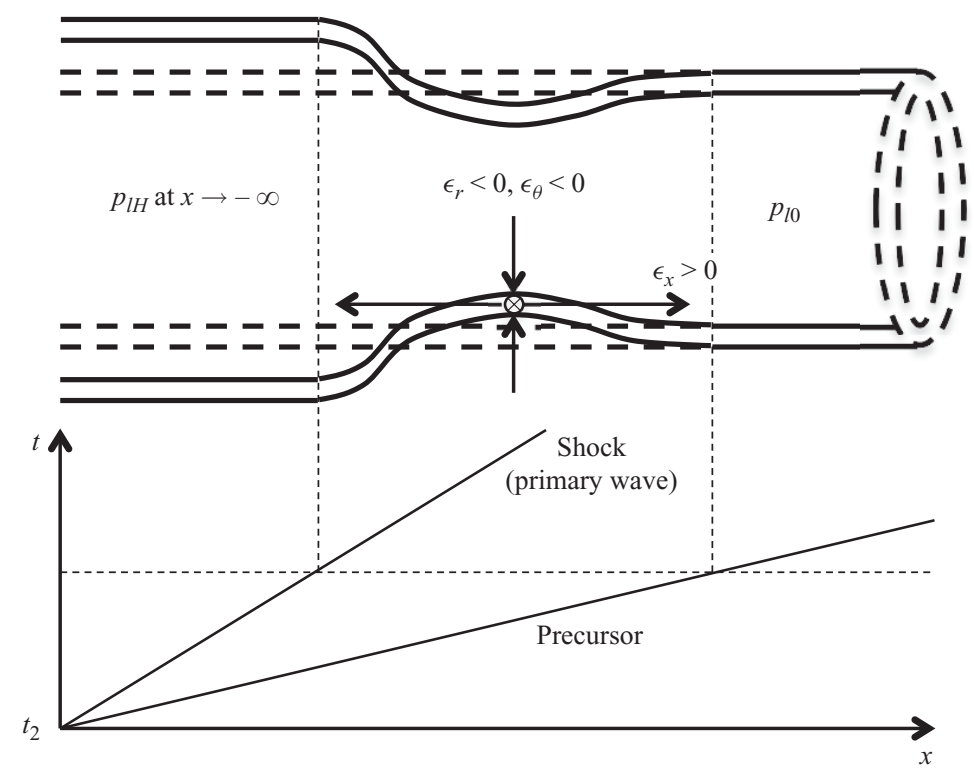

FIGURE 8. Illustration of the tube deformation and the corresponding $x-t$ diagram showing the relation of the precursory and primary waves.

strain measured at g1. It is seen that the precursory waves travel slightly faster than the sonic speed, $c_{s}$, of the tube material and are followed by the primary waves. The wave structure becomes more oscillatory as time progresses. Wave dispersion results from structural oscillations in which the tube dynamics come into play (Skalak 1956). Also, note that the precursory wavefront leads to contraction in the hoop direction, as illustrated in figure 8. Behind the shock front, the shock pressure expands the tube in the radial direction. As a result of mass (or volume) conservation, the section in front of the shock needs to elongate in the axial direction. The resulting stresses propagate with a precursory wave speed that is close to the sonic speed of the tube material. Since the precursor propagates faster than the shock, the separation between the precursory and shock fronts expands and the dispersion grows as the waves evolve 


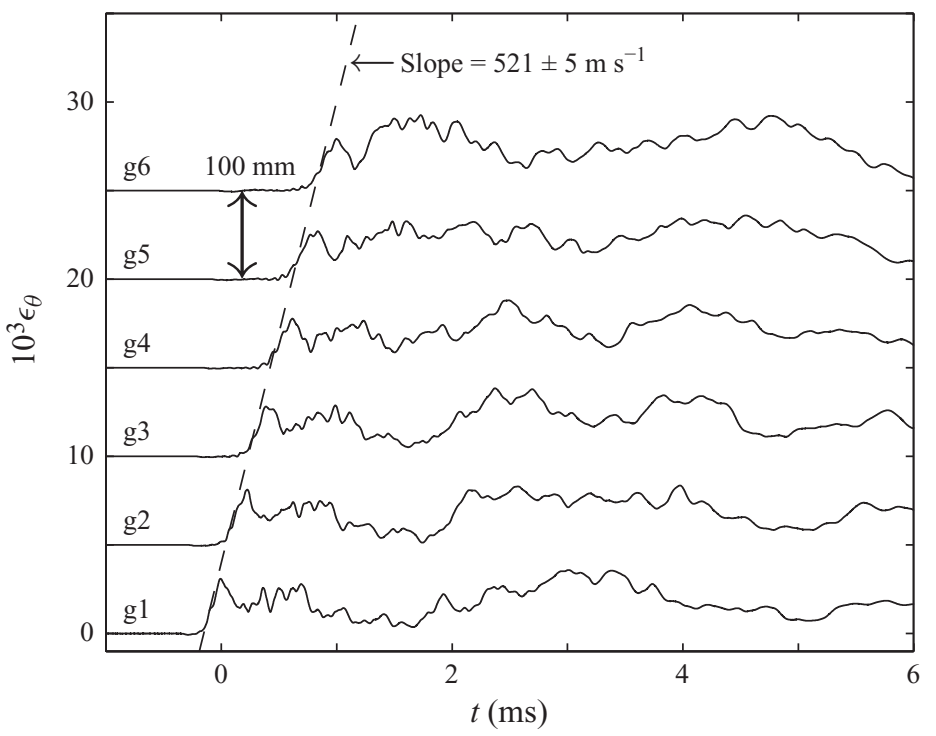

Figure 9. An example of the evolution of hoop strains for $H_{p}=2 \mathrm{~m}$ and $\alpha_{0}=0$. The slope of the dashed line is the computed wave speed.

(see figure 7). The net expansion in the radial direction behind the wavefront results in a tensile precursor and contraction in the axial direction because of the Poisson coupling between the axial and radial motions.

\subsection{Primary wave speeds}

We now examine the shock (or primary) waves, which produce larger amplitude hoop strains than the precursory waves. These water-hammer experiments are characterized by the drop height $H_{p}$ and the initial void fraction $\alpha_{0}$. To confirm repeatability in the measurements, three experimental runs were conducted for each case of $H_{p}$ and $\alpha_{0}$. In what follows, we choose some particular cases, and investigate the wave structures and infer the propagation speeds from the strain measurements.

The evolution of the hoop strains for the case of $H_{p}=2 \mathrm{~m}$ and $\alpha_{0}=0$ is shown in figure 9. Unlike in figures 6 and 7, the strain histories at the different gauges are separately presented in the vertical axis for clarity. Every strain gauge records the primary wave following a small-amplitude precursor. It also records a wave reflected from the tube bottom. In addition, as described in $\S 4.3$, the primary wave speed is computed and presented in figure 9; three different threshold strain levels $(30 \%$, $40 \%$ and $50 \%$ of the maximum strain measured at the strain gauge g1 before the reflected wave is observed) are chosen to determine the position of the wavefront. The computed speed $\left(521 \mathrm{~m} \mathrm{~s}^{-1}\right)$ is in reasonable agreement with the Korteweg-Joukowsky wave speed $\left(c_{l J}=518 \mathrm{~m} \mathrm{~s}^{-1}\right)$, and the dispersion due to the thresholding is very small. This suggests that the linear theory is effectively valid for the case of pure water, even though the wave is dispersive and unsteady.

The bubbly water case $\left(H_{p}=2 \mathrm{~m}, \alpha_{0}=0.0081\right)$ is presented in figure 10. A comparison of figures 9 and 10 reveals that the bubbles reduce the tube deformation. This is due to the fact that some fraction of the potential energy of the projectile is used to compress the bubbles. Moreover, the wave speed is remarkably reduced by the bubbles. It should also be pointed out that the propagation speed is fairly constant (with small standard deviation). To further see the effect of the bubbles, the case 


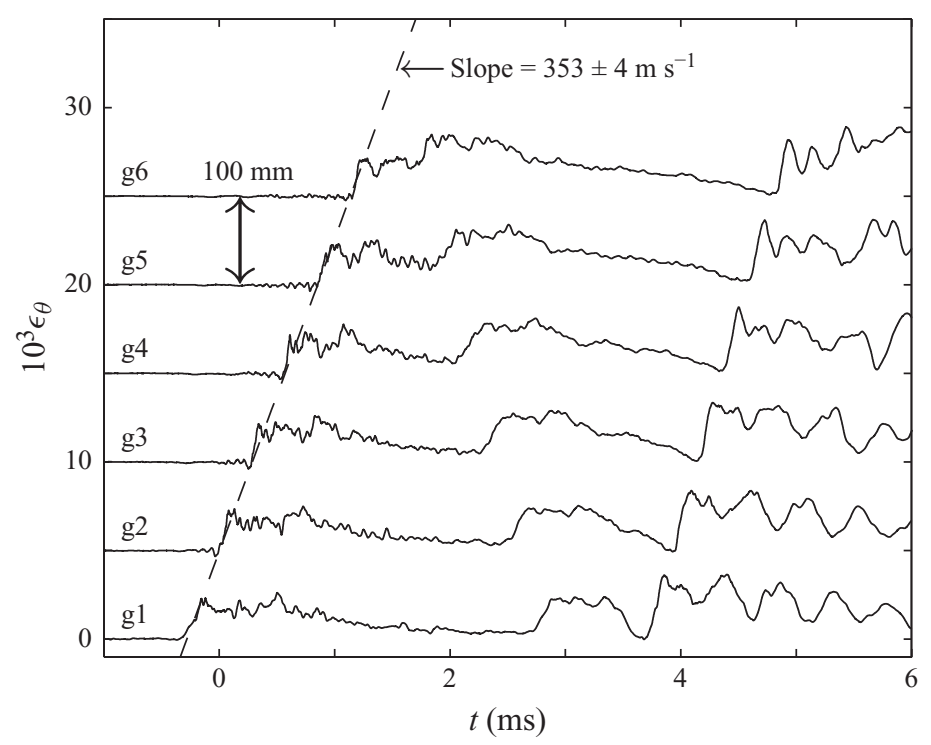

FIGURE 10. As figure 9, but with $H_{p}=2 \mathrm{~m}$ and $\alpha_{0}=0.0081$.

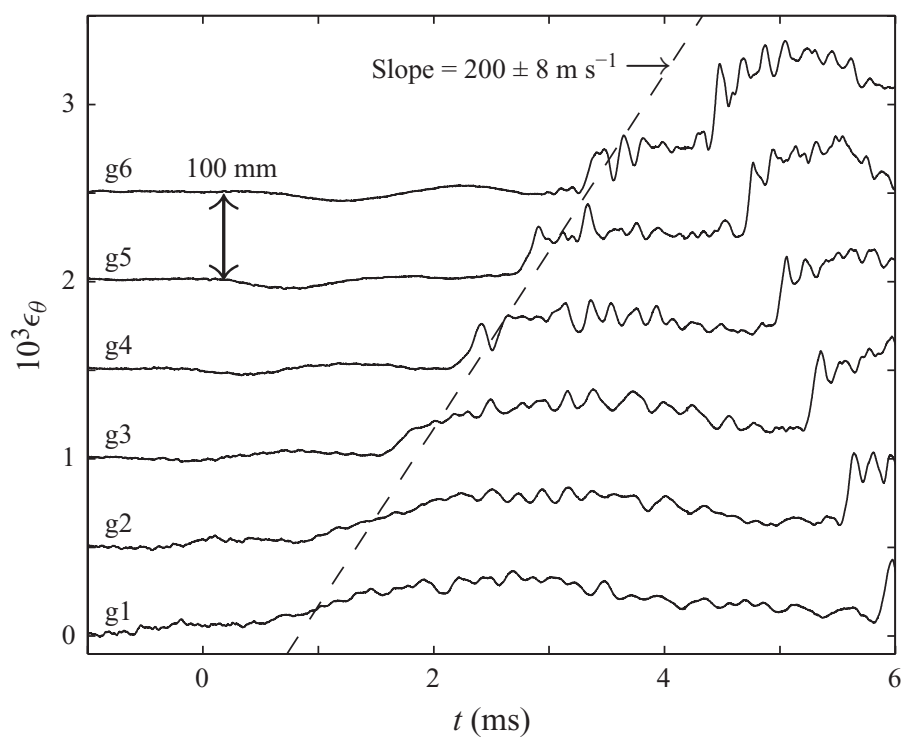

FIGURE 11. As figure 9, but with $H_{p}=0.5 \mathrm{~m}$ and $\alpha_{0}=0.0081$.

of the lower drop height $\left(H_{p}=0.5 \mathrm{~m}, \alpha_{0}=0.0081\right)$ is presented in figure 11 . In this case, the wave propagation is evidently unsteady in the sense that the compression wave steepens as it evolves. In figure 12 we superpose the shifted strain signals at different gauges from figures 10 and 11. This clearly confirms the steepening of the wavefront for the case of $H_{p}=0.5 \mathrm{~m}$. As pointed out in $\S 4.1$, the wave due to the air compression propagates before the primary wave; the larger-amplitude primary wave catches up the preceding wave so that the wavefront steepens as it evolves. As a result of the unsteadiness, the threshold value becomes more critical and the standard deviation of the computed wave speed becomes larger. It should be noticed 

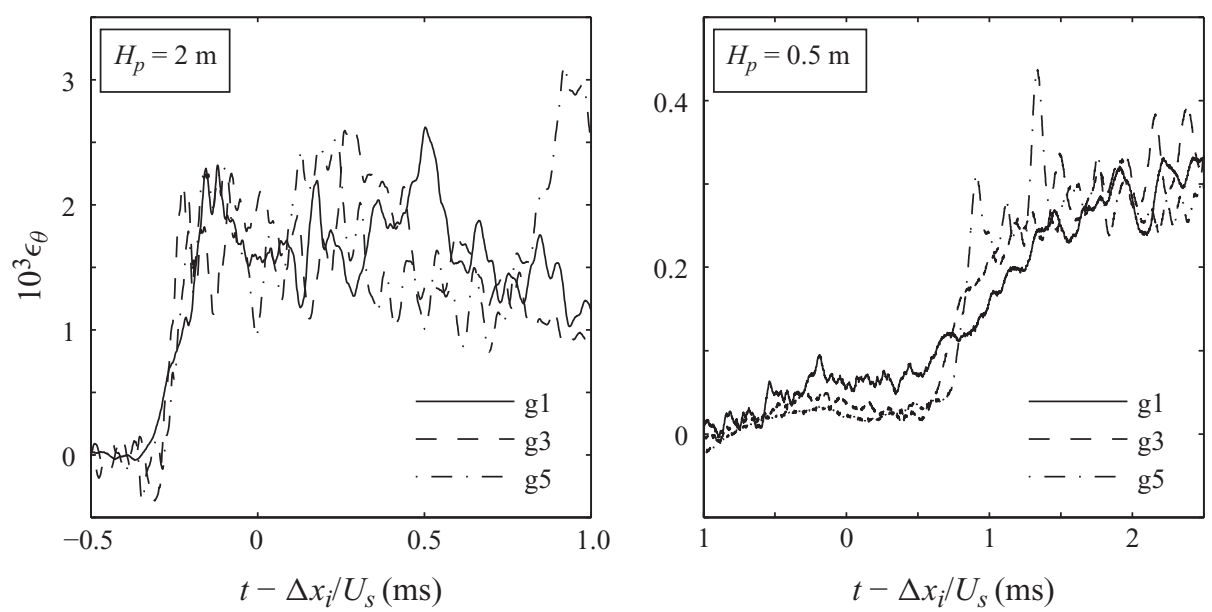

FiguRE 12. Superposition of the wave forms at different strain gauges in figures 10 and 11 , respectively. $U_{s}$ denotes the computed wave speeds and $\Delta x_{i}=0 \mathrm{~mm}, 200 \mathrm{~mm}$ and $400 \mathrm{~mm}$ are for $\mathrm{g} 1, \mathrm{~g} 3$ and $\mathrm{g} 5$, respectively.

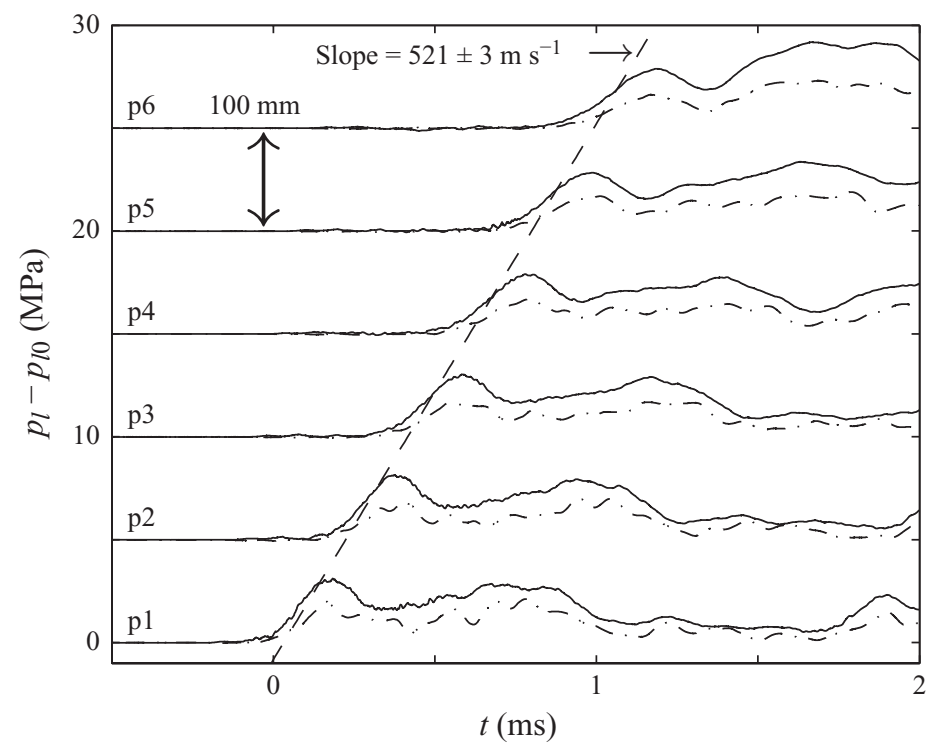

FigURE 13. An example of the evolution of liquid pressures for $H_{p}=2 \mathrm{~m}$ and $\alpha_{0}=0$. The solid and dash-dotted lines denote the measured and estimated pressures, respectively.

that the lower piston velocity (with decreasing $H_{p}$ ) further reduces the propagation speed. This is the effect of the gas-phase nonlinearity as pointed out in $\S 3.3$.

\subsection{Pressure measurements}

Measurements of the liquid pressure (as well as the hoop strain) along the tube wall were conducted in order to investigate their correlation. Here, we selected the two cases without air injection $\left(\alpha_{0}=0\right)$ and with bubbles $\left(\alpha_{0}=0.0024\right)$. For both cases, we chose the higher drop height of the projectile (i.e. $H_{p}=2 \mathrm{~m}$ ).

The evolution of the liquid pressure for the case of $\alpha_{0}=0$ is presented in figure 13 . For comparison, the pressure evolution estimated from the quasi-static relation (2.7) 


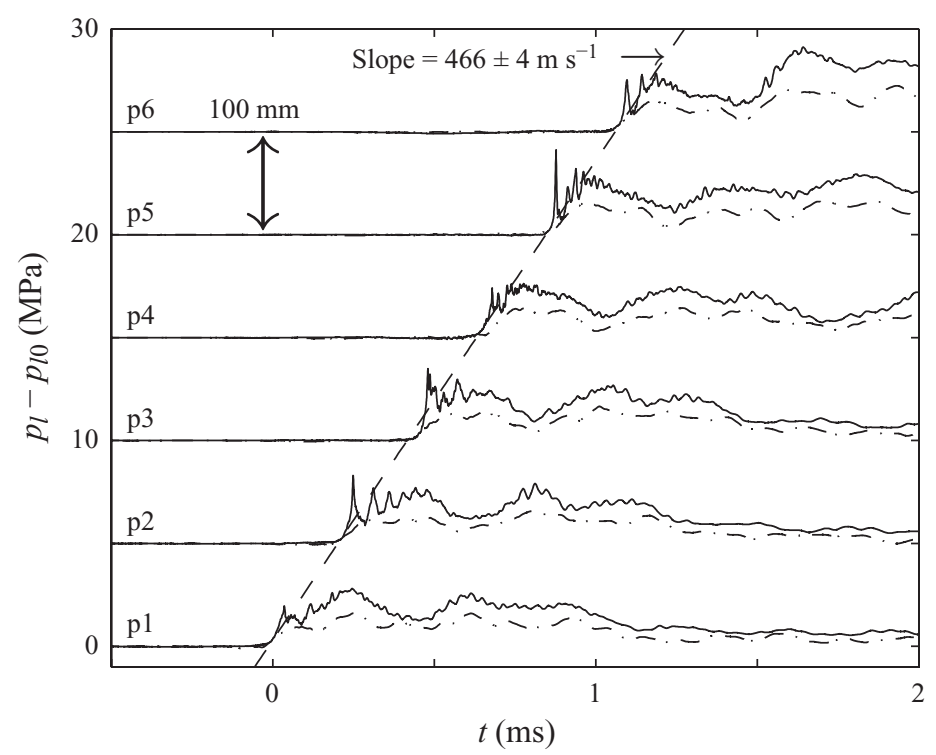

FIGURE 14. As figure 13, but with $\alpha_{0}=0.0024$.

given the measured hoop strains is also plotted. We observe a quantitative discrepancy between the measurement and the prediction. The wall thickness, across which a stress distribution exists, needs to be considered to accurately predict the fluid pressure from the hoop strains (Tijsseling 2007; Inaba \& Shepherd 2010). The neglect of the inertia and bending stiffness of the tube wall can also account for the discrepancy (Shepherd $\&$ Inaba 2009). It is also interesting to note that every pressure evolution shows a considerable drop after the first peak. After the buffer collides with the fluid surface, a compression wave would be generated and propagate in the buffer from the collision side. The generated shock will be reflected at the other end of the buffer as a tension wave, which will eventually be transmitted back to the fluid. The time for the two-way passage of waves in the buffer is about $0.4 \mathrm{~ms}$ and approximately consistent with the observation in figure 13.

Next, we examine the case with bubbles in figure 14. Now that the bubbles collapse after the shock compression, the pressure signals exhibit some oscillations due to radiated pressure pulses from the bubbles neighbouring the pressure transducers. It is obvious that the amplitude and frequency of the radiated pressure waves at the pressure transducers $\mathrm{p} 1-\mathrm{p} 6$ vary because the size and standoff distance of bubbles neighbouring the measurement points would be different. However, in this example, the structural response is rather insensitive to the bubble dynamics (see the pressure evolution estimated from the strain signals). To investigate the pressure radiation from collapsing bubbles, we consider an idealized, one-way-coupled flow in which spherical bubbles are forced according to a step-wise pressure change. The radiated pressure field is given by (Brennen 1995)

$$
p_{a}=\frac{\rho_{l} R}{r}\left(2 \dot{R}^{2}+R \ddot{R}\right),
$$

where $r$ is the distance from the bubble centre. To determine the bubble dynamics on the right-hand side of (4.1), we solve the equation of Gilmore (1952), which is a generalization of the Rayleigh-Plesset equation and accounts for effects of liquid 

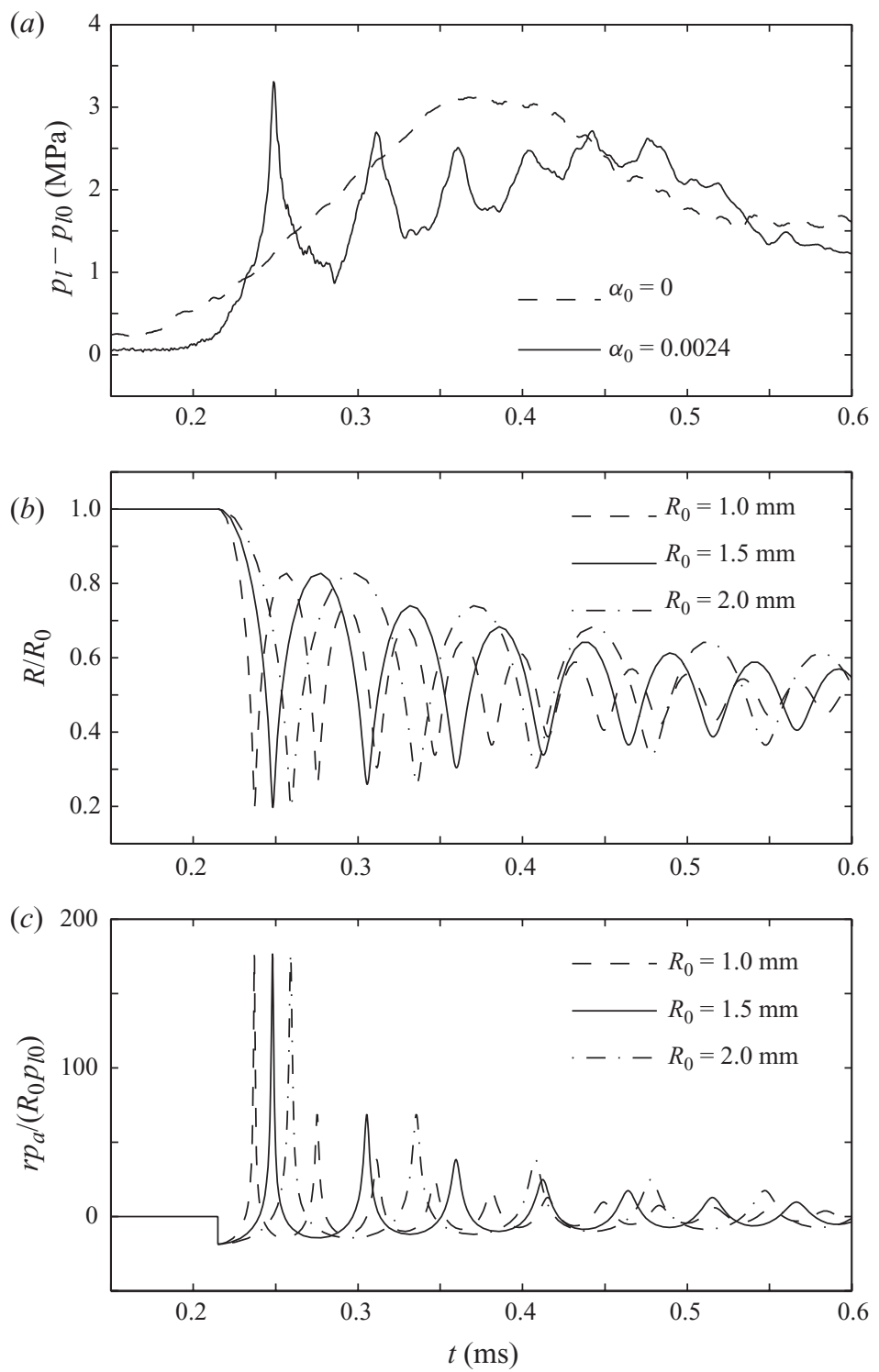

Figure 15. (a) Evolution of the liquid pressures at p2 in figures 13 and 14. For comparison, (b) the one-way-coupled Gilmore solution and $(c)$ the corresponding radiated pressure with a stepwise pressure change (one atmosphere to $2 \mathrm{MPa}$ ) at $0.215 \mathrm{~ms}$ are presented.

compressibility, with the assumption of adiabatic air and the effects of surface tension and vapour pressure (at $23^{\circ} \mathrm{C}$ ). As an example, in figure 15 we compare the pressure signals at p2 in figures 13 and 14 to the one-way-coupled flow computation in which the shock pressure is assumed to be $2 \mathrm{MPa}$ and the equilibrium bubble radius is inferred from the bubble images in $\S 4.2$. In this particular example, the time for bubble collapses with $R_{0}=1.5 \mathrm{~mm}$ and the associated radiated pressure pulses corresponds well to that for the observed sharp pressure peaks at p2.

As in figure 12, the shifted pressure signals at different measurement points from figures 13 and 14 are superposed in figures 16 and 17, respectively. The mean and 


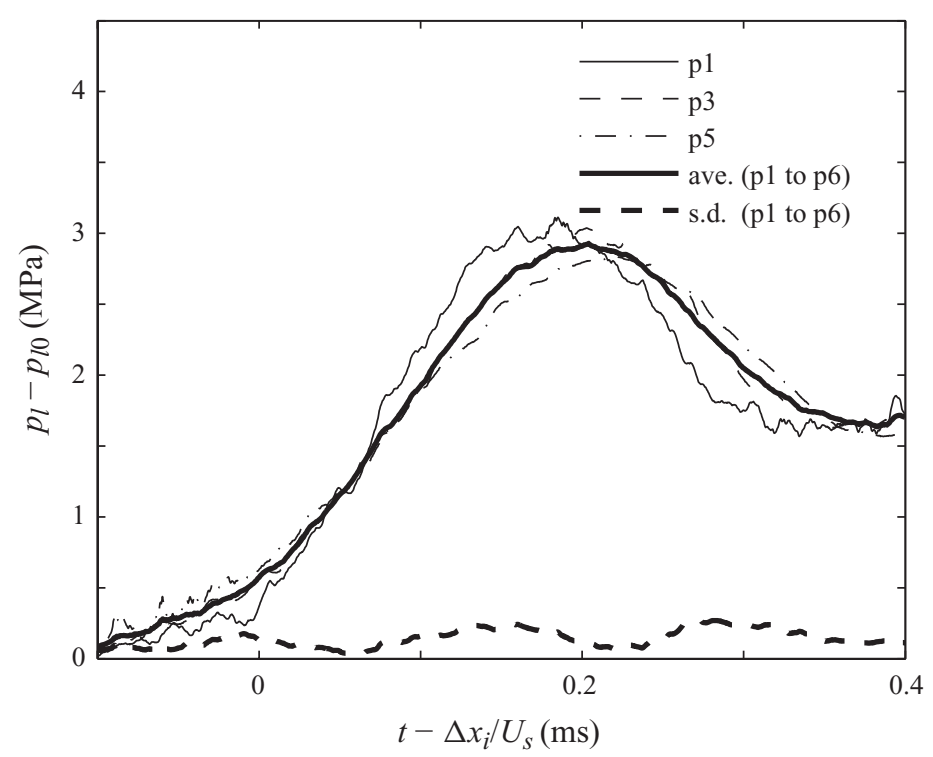

FIGURE 16. Superposition of the wave forms at different pressure transducers in figure 13 .

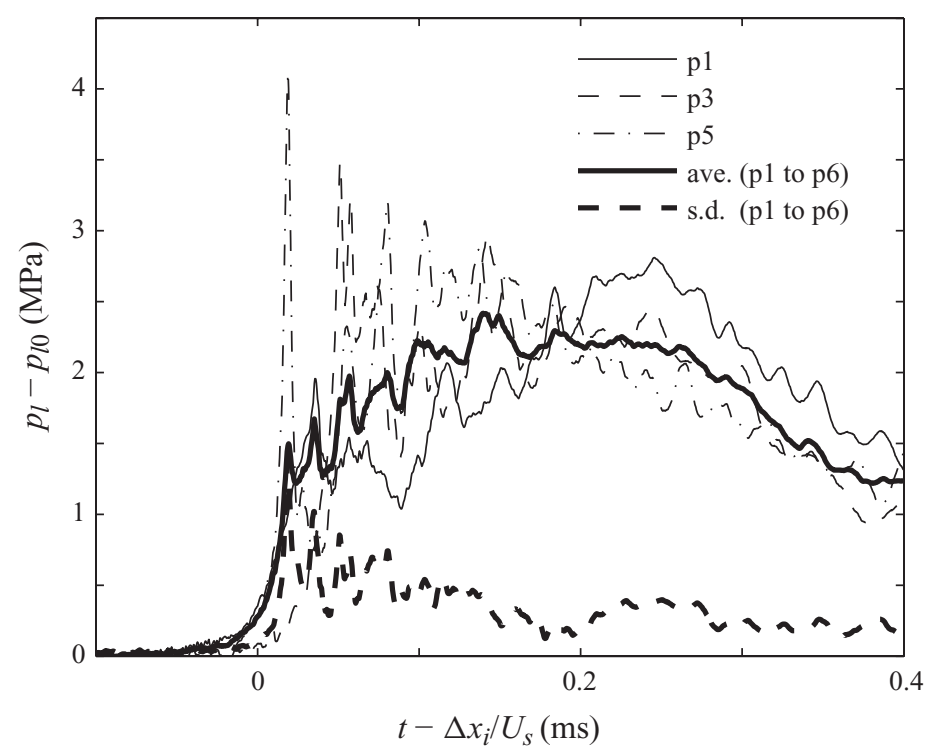

FIGURE 17. Superposition of the wave forms at different pressure transducers in figure 14.

standard deviation of the six different signals are also plotted. In the case of no bubbles, the pressure wave decays so gradually that the standard deviation of the different time frames is limited. With the air injection, on the contrary, the pressure signals are contaminated by a wave scattering due to the collapsing bubbles. As a result, the standard deviation is emphasized in particular just after the shock compression, but exhibits gradual decay as the bubble dynamics are damped away from the shock front. We also notice that the averaged pressure signal is rather smoothed because the individual fluctuations with different frequencies are cancelled 


\begin{tabular}{|c|c|c|c|c|c|c|}
\hline $\begin{array}{l}\alpha_{0}^{\text {mean }} \\
\Delta \alpha_{0}\end{array}$ & 0 & $\begin{array}{l}0.0013 \\
\pm 0.0001\end{array}$ & $\begin{array}{l}0.0024 \\
\pm 0.0001\end{array}$ & $\begin{array}{l}0.0056 \\
\pm 0.0001\end{array}$ & $\begin{array}{l}0.0081 \\
\pm 0.0001\end{array}$ & $\begin{array}{l}0.010 \\
\pm 0.001\end{array}$ \\
\hline$H_{p}=2 \mathrm{~m}$ & $\begin{array}{l}521 \pm 5 \\
524 \pm 2 \\
524 \pm 3\end{array}$ & $\begin{array}{l}502 \pm 1 \\
499 \pm 3 \\
492 \pm 2\end{array}$ & $\begin{array}{l}459 \pm 2 \\
458 \pm 1 \\
475 \pm 3\end{array}$ & $\begin{array}{l}404 \pm 2 \\
386 \pm 2 \\
388 \pm 1\end{array}$ & $\begin{array}{l}353 \pm 4 \\
356 \pm 2 \\
356 \pm 1\end{array}$ & $\begin{array}{l}313 \pm 2 \\
329 \pm 2 \\
321 \pm 2\end{array}$ \\
\hline$H_{p}=0.5 \mathrm{~m}$ & $\begin{array}{l}508 \pm 4 \\
524 \pm 4 \\
515 \pm 5\end{array}$ & $\begin{array}{l}477 \pm 5 \\
440 \pm 9 \\
446 \pm 13\end{array}$ & $\begin{array}{l}421 \pm 13 \\
385 \pm 3 \\
368 \pm 3\end{array}$ & $\begin{array}{l}259 \pm 3 \\
242 \pm 4 \\
257 \pm 2\end{array}$ & $\begin{array}{l}200 \pm 8 \\
201 \pm 13 \\
200 \pm 10\end{array}$ & $\begin{array}{l}168 \pm 5 \\
170 \pm 5 \\
183 \pm 2\end{array}$ \\
\hline $\begin{array}{l}c_{J}(\kappa=1.4) \\
c_{J}(\kappa=1)\end{array}$ & $\begin{array}{l}518 \\
518\end{array}$ & $\begin{array}{l}279 \\
246\end{array}$ & $\begin{array}{l}221 \\
191\end{array}$ & $\begin{array}{l}153 \\
131\end{array}$ & $\begin{array}{l}129 \\
110\end{array}$ & $\begin{array}{l}117 \\
99.4\end{array}$ \\
\hline
\end{tabular}

TABLE 1. Primary wave speeds (together with sonic speeds $\left.c_{J}\right)\left(\mathrm{m} \mathrm{s}^{-1}\right)$ for every experimental run with various $H_{p}$ and $\alpha_{0}$. The error bounds in the wave speeds are standard deviations.

out. Finally, we note that the rise time of the shock in figure 16 is about $0.3 \mathrm{~ms}$ and comparable to the natural period of bubbles with $R_{0}=1 \mathrm{~mm}$; the larger bubbles cannot respond to the pressure forcing and may thus have minor contributions to the mixture compressibility to determine the primary wave speed. For future use, we report that the peak of the averaged pressures for $\alpha_{0}=0$ and 0.0024 is $2.92 \mathrm{MPa}$ and $2.42 \mathrm{MPa}$, respectively.

\subsection{Summary}

The wave speeds are calculated for each experimental run and are organized, together with the sonic speeds (3.4) with adiabatic and isothermal compression of air bubbles, in table 1. To compute the sonic speed of the mixture, the adiabatic assumption with $\kappa=1.4$ may be more appropriate, for the relaxation time for thermal diffusion in air bubbles in the experiments may be comparable to or larger than the measurement period. For air bubbles with $R_{0}=1 \mathrm{~mm}$, the relaxation time is estimated as $R_{0}^{2} / \alpha_{T} \approx 40 \mathrm{~ms}$, where $\alpha_{T}$ is the thermal diffusivity. Note that the difference in the wave speeds among the three experimental runs is small. Also, note that the standard deviations of the wave speeds are small in particular for $H_{p}=2 \mathrm{~m}$, indicating constant propagation speeds. The wave speeds are not well predicted by the KortewegJoukowsky linear wave speed when the air is injected; for these cases, the measured wave speeds are much higher than the sonic speeds. More specifically, the deviation, due to the gas-phase nonlinearity, from the sonic speeds is augmented with increasing the piston velocity (or $H_{p}$ ). In the next section, we show that the shock theory predicts the wave speeds better.

\section{Comparison of theory and experiments}

\subsection{Buffer dynamics}

The discrepancy between the linear theory and the experiments clearly suggests a need to incorporate the gas-phase nonlinearity. Now, we compare the shock theory to the experiments. One way to close the steady shock relations is to assign the piston velocity $u_{H}$. The buffer velocity may be regarded as representative values of $u_{H}$. For every experimental run reported in table 1 , the buffer position $x_{b}$ (represented by the two lines with a $10 \mathrm{~mm}$ separation seen in figure 3) was recorded using a high-speed video camera (Phantom v7.3, Vision Research) with a recording rate of 32000 frames per second (f.p.s.), and the position history was extracted from the 


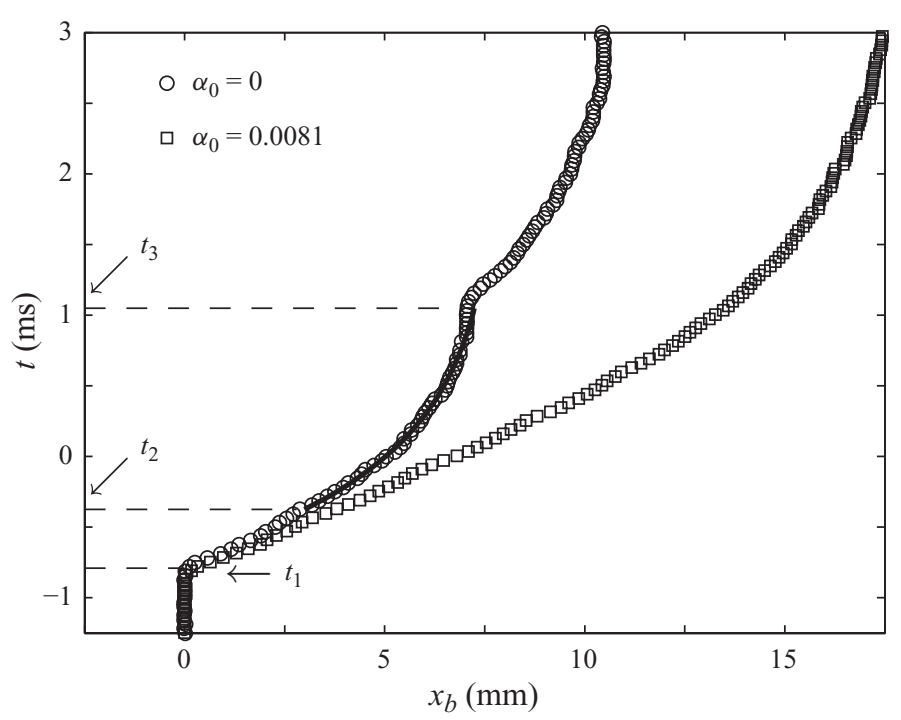

Figure 18. Evolution of the buffer positions for $H_{p}=2 \mathrm{~m}$. The solid line denotes an exponential fitted to the case of $\alpha_{0}=0$.

movies with MATLAB image processing. As an example, the evolution of the buffer position for the case of $H_{p}=2 \mathrm{~m}$ and $\alpha_{0}=0$ is exhibited in figure 18 . The projectile impacts the buffer at time $t_{1}$; the projectile and the buffer separate after the collision. The buffer then impacts the top of the fluid column at time $t_{2}$. The projectile again impacts the buffer at time $t_{3}$. For comparison, the case of $H_{p}=2 \mathrm{~m}$ and $\alpha_{0}=0.0081$ is also plotted in this figure. Because the reaction force from the fluid is reduced with (more compressible) bubbles, the buffer motion shows more gradual decay.

The buffer motion from $t_{2}$ to $t_{3}$ may be described by Newton's second law (Dashpande, Heaver \& Fleck 2006; Shepherd \& Inaba 2009). For simplicity, the buffer is treated as a rigid body and wall friction is neglected. The equation of motion of the buffer is then given by

$$
M_{b} \ddot{x}_{b}=-\Delta p_{l} A_{0},
$$

where $M_{b}$ is the mass of the buffer and the right-hand side represents the pressure force acting on the bottom of the buffer. In the linear case, this pressure force may be approximated by $\Delta p_{l} A_{0}=\rho_{0} c_{J} \dot{x}_{b} A_{0}$, as can be derived from (3.14) and (3.15). Integrating (5.1) once and specifying the initial condition at time $t_{2}$, we get a solution of the form

$$
\dot{x}_{b}=\dot{x}_{b}\left(t_{2}\right) \exp \left(-\frac{t-t_{2}}{\tau}\right),
$$

where $\tau$ is the relaxation time for the exponential decay:

$$
\tau=\frac{M_{b}}{\rho_{0} c_{J} A_{0}} .
$$

The measured buffer positions between $t_{2}$ and $t_{3}$ were fitted to an exponential by the least-squares method. In addition, the time $t_{2}$ was determined by observing the still images taken from the high-speed camera. The resulting buffer velocity $\dot{x}_{b}^{\text {fitted }}\left(t_{2}\right)$ and the relaxation time $\tau^{\text {fitted }}$ for every experimental run with $H_{p}=2 \mathrm{~m}$ are summarized in table 2 . In addition, the buffer velocity after the measurement period $t_{m}$ is estimated from the fitted function. Here, we define the measurement period for each run as 


\begin{tabular}{|c|c|c|c|c|c|c|c|}
\hline $\begin{array}{l}\alpha_{0}^{\text {mean }} \\
\Delta \alpha_{0}\end{array}$ & & 0 & $\begin{array}{l}0.0013 \\
\pm 0.0001\end{array}$ & $\begin{array}{l}0.0024 \\
\pm 0.0001\end{array}$ & $\begin{array}{l}0.0056 \\
\pm 0.0001\end{array}$ & $\begin{array}{l}0.0081 \\
\pm 0.0001\end{array}$ & $\begin{array}{l}0.010 \\
\pm 0.001\end{array}$ \\
\hline$\dot{x}_{b}^{\text {fitted }}\left(t_{2}\right)$ & $\left(\mathrm{m} \mathrm{s}^{-1}\right)$ & $\begin{array}{l}6.9 \\
6.9 \\
6.9\end{array}$ & $\begin{array}{l}8.5 \\
8.0 \\
7.9\end{array}$ & $\begin{array}{l}8.2 \\
8.3 \\
8.4\end{array}$ & $\begin{array}{l}8.4 \\
8.0 \\
8.2\end{array}$ & $\begin{array}{l}7.8 \\
8.5 \\
8.1\end{array}$ & $\begin{array}{l}7.3 \\
7.3 \\
7.9\end{array}$ \\
\hline$\dot{x}_{b}^{\text {fitted }}\left(t_{2}+t_{m}\right)$ & $\left(\mathrm{m} \mathrm{s}^{-1}\right)$ & $\begin{array}{l}2.2 \\
2.0 \\
1.8\end{array}$ & $\begin{array}{l}3.1 \\
3.5 \\
3.4\end{array}$ & $\begin{array}{l}3.6 \\
3.3 \\
3.5\end{array}$ & $\begin{array}{l}3.5 \\
3.2 \\
3.7\end{array}$ & $\begin{array}{l}3.8 \\
4.2 \\
3.6\end{array}$ & $\begin{array}{l}3.2 \\
3.3 \\
3.3\end{array}$ \\
\hline$\tau^{\text {fitted }}$ & $(\mathrm{ms})$ & $\begin{array}{l}0.82 \\
0.76 \\
0.71\end{array}$ & $\begin{array}{l}1.0 \\
1.2 \\
1.2\end{array}$ & $\begin{array}{l}1.3 \\
1.2 \\
1.2\end{array}$ & $\begin{array}{l}1.4 \\
1.4 \\
1.6\end{array}$ & $\begin{array}{l}2.0 \\
2.0 \\
2.0\end{array}$ & $\begin{array}{l}1.8 \\
1.8 \\
1.8\end{array}$ \\
\hline $\begin{array}{l}\tau(\kappa=1.4) \\
\tau(\kappa=1)\end{array}$ & $\begin{array}{l}(\mathrm{ms}) \\
(\mathrm{ms})\end{array}$ & $\begin{array}{l}0.713 \\
0.713\end{array}$ & $\begin{array}{l}1.33 \\
1.50\end{array}$ & $\begin{array}{l}1.68 \\
1.93\end{array}$ & $\begin{array}{l}2.43 \\
2.84\end{array}$ & $\begin{array}{l}2.89 \\
3.39\end{array}$ & $\begin{array}{l}3.19 \\
3.75\end{array}$ \\
\hline
\end{tabular}

TABLE 2. Velocity and relaxation time of the buffer motion for every experimental run with $H_{p}=2 \mathrm{~m}$. For comparison, the relaxation time is calculated from the linear analysis.

$t_{m}=\left(x_{g 6}-x_{g 1}\right) / U_{s}$, where $x_{g 6}-x_{g 1}=500 \mathrm{~mm}$ and the wave speed $U_{s}$ is taken from table 1. It turns out that the constant buffer velocity, which the shock theory favours, is not well achieved in the measurements. Nonetheless, the measured waves propagate fairly with constant speeds (see for example the wave propagation in figure 10), regardless of the decelerating buffer motion. This may be explained as follows. The rarefaction wave resulting from the deceleration cannot catch up the preceding wave that is propagating faster with larger peak pressure. As a result, the peak pressure of the wavefront will not change if the effects of damping and tube inertia are minimal (see figure 16). Assuming that the peak pressure is critical to the shock state (or the corresponding shock speed) even in the case with bubbles, we will obtain constant propagation speeds as observed in the experiments. Even though the induced velocity corresponding to the peak pressure is unknown, it may be bounded by the initial and decaying buffer velocities. That is, we may consider $\dot{x}_{b}^{\text {fitted }}\left(t_{2}\right)$ and $\dot{x}_{b}^{\text {fitted }}\left(t_{2}+t_{m}\right)$ as the upper and lower bounds, respectively, of the representative value of $u_{H}$.

\subsection{Induced fluid motion}

Provided that the slip between the host liquid and the bubbles is minimal, it is reasonable to trace the positions of the compressed bubbles, rather than the buffer positions, in the sequential images (as presented in figure 4) in order to infer the induced velocity. Here, the cross-correlation technique with MATLAB is adopted to compute the translation of the compressed bubbles between the two images with time separation $\Delta t$.

The procedure for $H_{p}=2 \mathrm{~m}$ and $\alpha_{0}=0.0013$ is illustrated in figure 19. First, we set the sub-image in the frame at $t$ to enclose the collapsing bubbles behind the shock front, and calculate its cross-correlation, which is a function of the offset in both the axial and radial directions, with respect to the (reference) frame at $t+\Delta t$. Then, the offset that yields the peak of the cross-correlation can be regarded as the displacement of the bubbles during $\Delta t$. If taking $\Delta t=0.5 \mathrm{~ms}$, the mean particle velocity is estimated as $\Delta x / \Delta t=7.35 \pm 0.67 \mathrm{~m} \mathrm{~s}^{-1}$, where $\Delta x$ is the displacement in the axial direction and the deviation is due to one-pixel errors. Note that the bubbles seem to advect uniformly in space (i.e. the cross-correlation is sharply peaked), so that the velocity variation may be minor inside the sub-image. This indicates that 


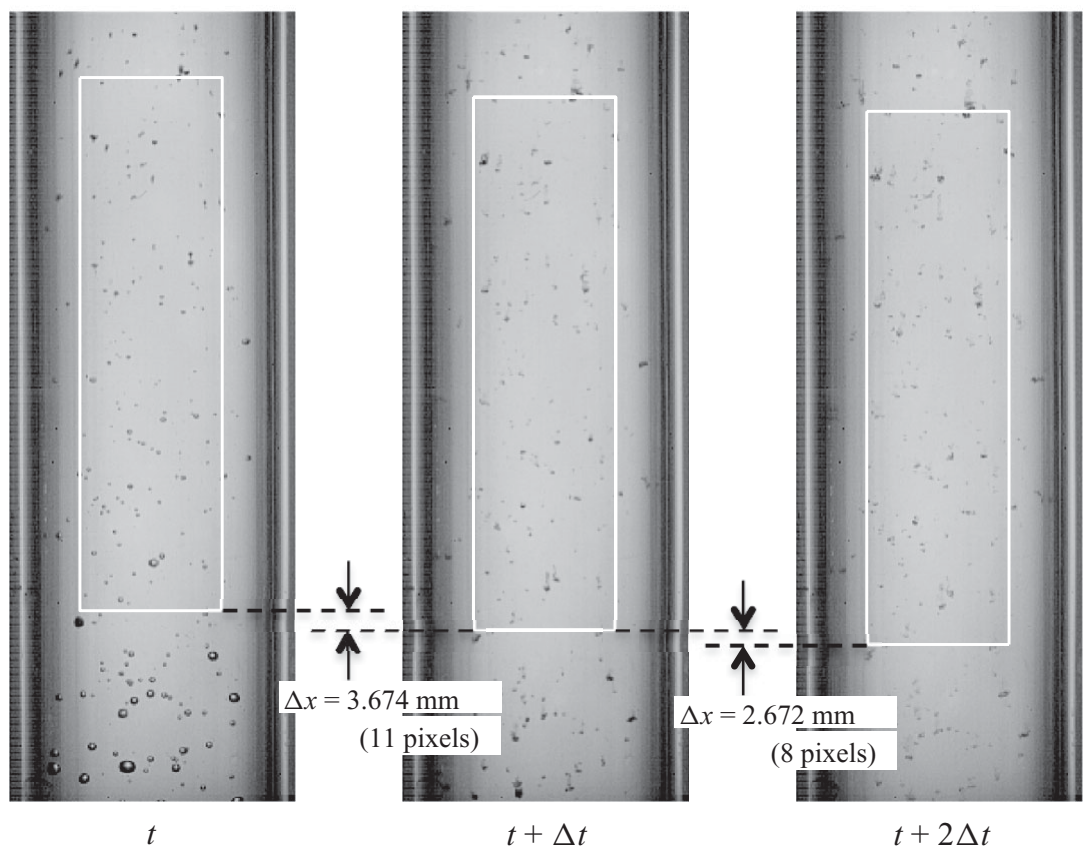

FIGURE 19. Illustration of the cross-correlation method to estimate the displacement of the compressed bubbles (encircled by the white line) that advect downward with the shock-induced ambient liquid flow for $H_{p}=2 \mathrm{~m}$ and $\alpha_{0}=0.0013$. The time separation, $\Delta t$, is $0.5 \mathrm{~ms}$.

the typical wavelength may be large enough to minimize the relative motion of the bubbles (Brennen 1995). Repeating the same procedure between the frames at $t+\Delta t$ and $t+2 \Delta t$, the mean velocity is now computed as $\Delta x / \Delta t=5.34 \pm 0.67 \mathrm{~m} \mathrm{~s}^{-1}$. That is, like the buffer motion, the induced fluid motion will considerably decay within the measurement period $\left(t_{m} \approx 1 \mathrm{~ms}\right)$. We also confirm that these estimated values fall between the upper and lower limits in table 2 .

\subsection{Theoretical predictions}

Finally, we compare the theoretical shock speeds with the measured speeds for $H_{p}=2 \mathrm{~m}$. As discussed in $\S 5.1, \dot{x}_{b}^{\text {fitted }}\left(t_{2}\right)$ and $\dot{x}_{b}^{\text {fitted }}\left(t_{2}+t_{m}\right)$ in table 2 are regarded as the upper and lower bounds of $u_{H}$ and used to close the steady shock relations. To be specific, we select the maximum of $\dot{x}_{b}^{\text {fitted }}\left(t_{2}\right)$ and the minimum of $\dot{x}_{b}^{\text {fitted }}\left(t_{2}+t_{m}\right)$ (out of the three experimental runs) for each $\alpha_{0}$ in order to define estimation errors. We consider only the case of isothermal compression $(\kappa=1)$ of bubbles and neglect the vapour pressure and the surface tension (i.e. $p_{v}=0, \Upsilon=0$ ) for the computations; variations due to these parameters are much smaller than those due to the range of $u_{H}$.

In figure 20, the theoretical shock speeds are compared to the measured values for $H_{p}=2 \mathrm{~m}$. The errors in the measured speeds and void fractions (documented in table 1) are small and omitted for clarity. We confirm that the value of $u_{H}$ is irrelevant at $\alpha_{0}=0$ since the liquid-phase nonlinearity is minor in this example. However, the measured speeds for the cases with air injection clearly exhibit differences from the sonic speeds, and clearly indicate the effect of the gas-phase nonlinearity on the wave speeds. Although the uncertainty associated with the non-constant value of $u_{H}$ increases as the void fraction increases, the shock theory is capable of more accurately capturing the trend with increasing $\alpha_{0}$ than the linear theory. This 


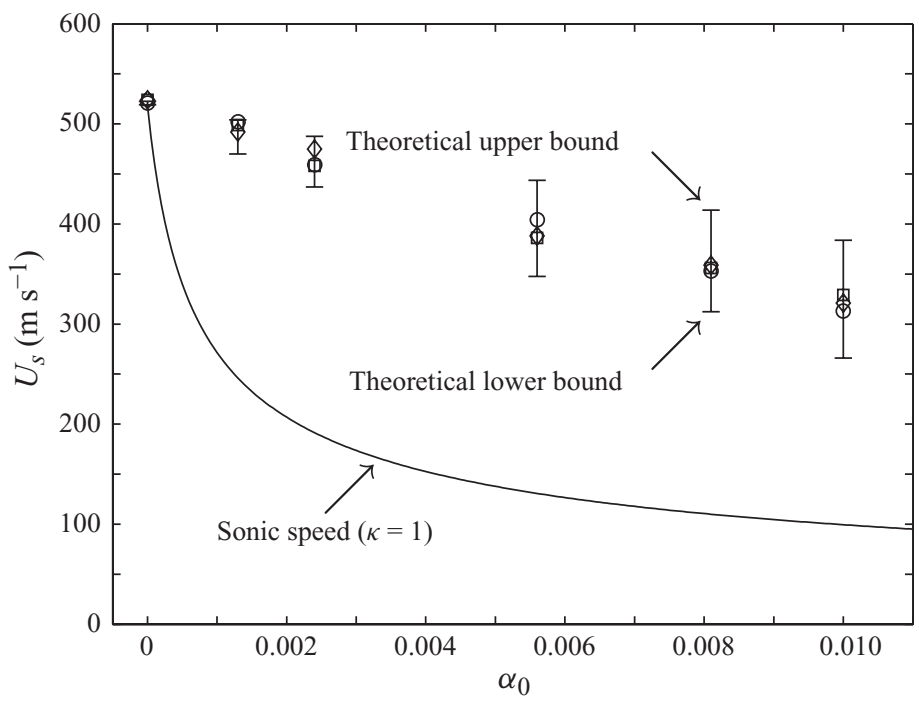

FIGURE 20. Estimated wave speeds as a function of $\alpha_{0}$. The symbols denote the measurements for every experimental run with $H_{p}=2 \mathrm{~m}$.

comparison suggests that the gas-phase nonlinearity (as well as FSI) needs to be considered to properly estimate the propagation speeds of finite-amplitude waves in a mixture-filled pipe. Finally, we note that the averaged peak pressures, $p_{l H}$, for $\alpha_{0}=0$ and 0.0024 (presented in $\S 4.5$ ) yield $u_{H}=5.44 \mathrm{~m} \mathrm{~s}^{-1}$ and $5.04 \mathrm{~m} \mathrm{~s}^{-1}$, respectively, which fall between the upper and lower limits of the buffer velocities.

To quantify the decay rate as well as the dispersion, the dynamics of both fluids and structures need to be evaluated. The viscoelasticity of the polycarbonate may affect the wave speed and damping (Meißner \& Frank 1977; Gally, Güney \& Rieutord 1979; Suo \& Wylie 1990; Covas et al. 2004). The unsteady wall friction may also have some impact on the wave damping (Bergant 2001). If bubble fission occurs after passage of the shock, the fission damping needs to be included in bubbledynamic modelling (Brennen 2002). Moreover, because polydispersity results in different frequency responses for different-sized bubbles, phase cancellations cause an additional apparent damping of the wave propagation (Smereka 2002; Colonius et al. 2008; Ando, Colonius \& Brennen 2009; Ando 2010).

\section{Conclusions}

A quasi-one-dimensional conservation law governing continuum bubbly flows in a deformable cylindrical tube was formulated and used to derive steady shock relations. The modified bulk modulus of the mixture is introduced, and the nonlinear effect due to the gas-phase compressibility is shown to be important for shock propagation. The present FSI shock theory is found to be in better agreement with the measured wave speeds than the linear theory. This suggests that the gas-phase nonlinearity (as well as FSI) needs to be taken into account to accurately estimate the propagation speeds of finite-amplitude waves in a bubbly-liquid-filled pipe.

The authors would like to express their thanks to T. Nishiyama for his help with the experimentation, R. Porowski for the bubble images and S. Hori for his observations about the experimental data. This work was supported by ONR grant N00014-06-1-0730. 


\section{REFERENCES}

ANDO, K. 2010 Effects of polydispersity in bubbly flows. PhD thesis, California Institute of Technology. Available at: http://thesis.library.caltech.edu/5859/.

Ando, K., Colonius, T. \& Brennen, C. E. 2009 Improvement of acoustic theory of ultrasonic waves in dilute bubbly liquids. J. Acoust. Soc. Am. 126, EL69-EL74.

Bergant, A. 2001 Developments in unsteady pipe flow friction modeling. J. Hydraul. Res. 39, $249-257$.

BEYLICH, A. E. \& GüLHAN, A. 1990 On the structure of nonlinear waves in liquids with gas bubbles. Phys. Fluids A 2, 1412-1428.

Brennen, C. E. 1994 Hydrodynamics of Pumps. Oxford University Press.

Brennen, C. E. 1995 Cavitation and Bubble Dynamics. Oxford University Press.

Brennen, C. E. 2002 Fission of collapsing cavitation bubbles. J. Fluid Mech. 472, 153-166.

Brennen, C. E. 2005 Fundamentals of Multiphase Flow. Cambridge University Press.

Campbell, I. J. \& Pitcher, A. S. 1958 Shock waves in a liquid containing gas bubbles. Proc. $R$. Soc. Lond. A 243, 534-545.

Cole, R. H. 1948 Underwater Explosions. Princeton University Press.

Colonius, T., Hagmeijer, R., Ando, K. \& Brennen, C. E. 2008 Statistical equilibrium of bubble oscillations in dilute bubbly flows. Phys. Fluids 20, 040902.

Covas, D., Stoianov, I., Mano, J., Romas, H., Graham, N. \& Maksimovic, C. 2004 The dynamic effect of pipe-wall viscoelasticity in hydraulic transients. Part 1. Experimental analysis and creep characterization. J. Hydraul. Res. 42, 516-530.

Dashpande, V. S., Heaver, A. \& Fleck, N. A. 2006 An underwater shock simulator. Proc. R. Soc. Lond. A 462, 1021-1041.

Delale, C. F., Nas, S. \& Tryggvason, G. 2005 Direct numerical simulations of shock propagation in bubbly liquids. Phys. Fluids 17, 121705.

Delale, C. F. \& Tryggvason, G. 2008 Shock structure in bubbly liquids: comparison of direct numerical simulations and model equations. Shock Waves 17, 433-440.

Gally, M., GüNeY, M. \& Rieutord, E. 1979 An investigation of pressure transients in viscoelastic pipes. J. Fluids Engng 101, 495-499.

Ghidaoui, M. S., Zhao, M., McInnis, D. A. \& Axworthy, D. H. 2005 A review of water hammer theory and practice. Appl. Mech. Rev. 58, 49-76.

GiLmore, F. R. 1952 The collapse and growth of a spherical bubble in a viscous compressible liquid. Hydrodyn. Lab. Rep. 26-4. California Institute of Technology.

InABA, K. \& Shepherd, J. E. 2010 Flexural waves in fluid-filled tubes subject to axial impact. J. Press. Vessel Technol. 132, 021302.

Johnsen, E. \& Colonius, T. 2009 Numerical simulations of non-spherical bubble collapse. J. Fluid Mech. 629, 231-262.

Joukowsky, N. E. 1898 Memoirs of the Imperial Academy Society of St. Petersburg. Proc. Am. Water Works Assoc. 24, 341-424.

Kameda, M. \& Matsumoto, Y. 1996 Shock waves in a liquid containing small gas bubbles. Phys. Fluids 8, 322-335.

Kameda, M., Shimaura, N., Higashino, F. \& Matsumoto, Y. 1998 Shock waves in a uniform bubbly flow. Phys. Fluids 10, 2661-2668.

Kobori, T., Yokoyama, S. \& MiYashiro, H. 1955 Propagation velocity of pressure wave in pipe line. Hitachi Hyoron 37, 33-37.

KorteWEG, D. J. 1878 Ueber die Fortpflanzungsgeschwindigkeit des Schalles in elastischen Röhren. Ann. Phys. Chem. 5, 525-542.

Kuznetsov, V. V., Nakoryakov, V. E., Pokusaev, B. G. \& Shreiber, I. R. 1978 Propagation of perturbations in a gas-liquid mixture. J. Fluid Mech. 85, 85-96.

MeISSNER, E. \& Frank, P.-G. 1977 Influence of pipe material on the damping of water hammer. In Proc. 17th Congress of the International Association for Hydraulic Research, Baden-Baden, Germany.

Nagayama, K., Mori, Y. \& Shimada, K. 2002 Shock Hugoniot compression curve for water up to 1 GPa by using a compressed gas gun. J. Appl. Phys. 91, 476-482.

Nigmatulin, R. I., Khabeev, N. S. \& Hai, Z. N. 1988 Waves in liquids with vapour bubbles. J. Fluid Mech. 186, 85-117. 
NoORdZIJ, L. \& VAN WiJngaARden, L. 1974 Relaxation effects, caused by relative motion, on shock waves in gas-bubble/liquid mixtures. J. Fluid Mech. 66, 115-143.

Pedley, T. J. 1980 The Fluid Mechanics of Large Blood Vessels. Cambridge University Press.

ShePherd, J. E. \& InABA, K. 2009 Shock loading and failure of fluid-filled tubular structures. In Dynamic Failure of Materials and Structures (ed. A. Shukla, G. Ravichandran \& Y. D. S. Rajapakse). Springer.

SkalaK, R. 1956 An extension of the theory of water hammer. Trans. ASME 78, 105-116.

SMereKa, P. 2002 A Vlasov equation for pressure wave propagation in bubbly fluids. J. Fluid Mech. 454, 287-325.

Suo, L. \& Wylie, E. B. 1990 Complex wavespeed and hydraulic transients in viscoelastic pipes. J. Fluids Engng 112, 496-500.

Thompson, P. A. 1972 Compressible Fluid Dynamics. McGraw-Hill.

TiJsseling, A. S. 1996 Fluid-structure interaction in liquid-filled pipe systems: a review. J. Fluids Struct. 10, 109-146.

TiJsseling, A. S. 2007 Water hammer with fluid-structure interaction in thick-walled pipes. Comput. Struct. 85, 844-851.

Tijsseling, A. S., Lambert, M. F., Simpson, A. R., Stephens, M. L., VítKovský, J. P. \& Bergant, A. 2008 Skalak's extended theory of water hammer. J. Sound Vib. 310, 718-728.

Watanabe, M. \& Prosperetti, A. 1994 Shock waves in dilute bubbly liquids. J. Fluid Mech. 274, 349-381.

VAN WiJngaARDEN, L. 1968 On the equations of motion for mixtures of liquid and gas bubbles. J. Fluid Mech. 33, 465-474.

van WijngaARden, L. 1972 One-dimensional flow of liquids containing small gas bubbles. Annu. Rev. Fluid Mech. 4, 369-396.

Wylie, E. B. \& Streeter, V. L. 1993 Fluid Transients in Systems. Prentice Hall.

Zhang, Z. D. \& Prosperetti, A. 1994 Ensemble-averaged equations for bubbly flows. Phys. Fluids 6, 2956-2970. 